\title{
Ultra-Fast Current Differential Protection with High-Sensitivity for HVDC Transmission Lines
}

Haijin Liu ${ }^{\mathrm{a}}$, Bin Li ${ }^{\mathrm{a}}$, Weijie Wen ${ }^{\mathrm{a}}$, Timothy C. Green ${ }^{\mathrm{b}}$, Jihang Zhang ${ }^{\mathrm{a}}$, Ning Zhang ${ }^{\mathrm{c}}$ and Longlong Chen ${ }^{\mathrm{c}}$

a, Key Laboratory of Smart Grid of Ministry of Education, Tianjin University, Tianjin 300072, China.

b, the Department of Electrical and Electronic Engineering, Imperial College London, London SW7 2AZ, UK

c, Global Energy Interconnection Research Institute Co.Ltd, State Grid, Beijing 10084, China

Corresponding author: Weijie Wen, Email: weijie.wen@tju.edu.cn, Postal address: key Laboratory of Smart Grid of Ministry of Education, Tianjin University, Tianjin 300072, China.

\section{HIGHLIGHTS:}

- The intrinsic properties of the HVDC transmission line are analyzed.

- The fundamental relationship between protection performance and transmission line model is revealed.

- An ultra-fast current differential protection with high-sensitivity for HVDC transmission lines, integrated with function of fault detection and faulty pole selection, is proposed.

- The performance of the proposed protection method is verified.

\begin{abstract}
:
Protection of transmission lines is the most important defense against faults in multi-terminal HVDC system. Up until now, no single protection can balance the operation speed and sensitivity needed for all fault types. To solve this problem, a novel current differential protection for HVDC transmission line is proposed in this paper. The intrinsic properties of a HVDC transmission line are analyzed first. Then, essence of ideal current differential protection is revealed and fundamental relations between line models and performance degradation of existing differential protections are discussed. Based on this analysis, a novel current differential protection scheme, including fault detection and faulty pole selection, is developed, and Bergeron model is used to describe the electrical wave propagation process along line, resulting in only simple computations are needed in each calculation cycle. Finally, a simulation model of Wudongde $\pm 800 \mathrm{kV}$ HVDC project in China is established in PSCAD/EMTDC for verification. By discussing diversified faults and transient processes, simulation results prove that regardless of fault type, distance and transition impedance, the proposed differential protection can respond correctly. Main contribution of this paper is reducing the operation time of differential protection from hundreds of milliseconds to several milliseconds.
\end{abstract}

KEYWORDS: HVDC transmission lines, current differential protection, Bergeron model, fault detection and faulty pole selection. 


\section{Introduction}

With remarkable advantages of low operating losses, large transmission capacity and flexible power control, voltage source converter (VSC) or line-commutated converter (LCC) based high voltage direct current (HVDC) power system deployed on overhead transmission lines is a promising prospect for long-distance, large-capability power transmission and renewable power integration [1-3]. Because the long overhead transmission line is exposed to a complex environment, the probability of faults occurring is quite high. A protection scheme is essential for defense against faults on transmission lines to ensure the safety and stability of the whole power system $[4,5]$.

Examining existing projects, the feasible protection scheme for HVDC transmission lines uses a combination of three protection methods: traveling-wave protection, voltage derivative protection and conventional current differential protection[4,6,7]. The combination of different methods is needed to ensure that all types of faults could be detected and responded appropriately.[8]. To reduce the block time as much as possible or even completely avoid the block of VSC triggered by overcurrent, protection of transmission line should respond to large-current faults within a few milliseconds [9], but there is no need to respond to small-current faults so fast. To achieve a short response time under large-current faults, travelling-wave protection and voltage derivative protection are used as dual primary protections [10-12]. But these two protection methods are sensitive to transition impedance at fault point and might fail to respond to small-current faults, such as high-resistance faults [13]. Therefore, conventional current differential protection is integrated as backup protection [14], and its response time is hundreds of milliseconds. In short, no single existing protection method can provide performances of high-sensitivity and fast-operation speed for all fault types, resulting in two or more protection methods must be cooperated with each other in an overall protection scheme.

To simplify the protection scheme for HVDC transmission line, a promising approach is to improve the performance of differential protection so that it could respond to all internal faults within a few milliseconds and refuse to response to all external faults and system disturbance conditions, resulting in no requirement for other protection methods with high sampling frequency. Many scholars have made progress in this direction, and literature reviews are listed as follows.

In conventional current differential protection $[6,15,16]$, without special consideration of the transmission line model, the differential current is calculated by using measured currents directly, and delay time of $500 \mathrm{~ms}$ and block time of $600 \mathrm{~ms}$ is set to prevent mal-operation giving a total response time of $1.1 \mathrm{~s}$. With distributed capacitive current commonly regarded as the reason for mal-operation of conventional differential protection, in reference [9], a distributed parameter line model was used to calculate the distributed capacitive current for compensation so that delay time and block time are unnecessary and current differential protection with a response time of $10 \sim 20 \mathrm{~ms}$ is achieved. In reference [5,6,17], a frequency-dependent line model was used to calculate the differential current based on Time-Frequency domain transformation of measured signals in a time window of $2 \mathrm{~ms}$. With suitable protection criteria, sensitivity was ensured, but the relatively long calculation time results in a response time of 10 20ms. In reference $[7,15,18]$, based on polar-mode transformation and Bergeron line model, differential current was calculated, and the shortcoming was that for pole to ground faults, a false trip of protection on the healthy pole might occur.

It is clear from the literature that different transmission line models have been be used in differential protection but the fundamental relationship between line model and protection performance has never been revealed clearly, and response time of existing differential protection varies from 10ms to 1.1s and it is too long for HVDC system consisting of VSC. To further tackle this problem, this paper carried out analysis on the relation between line model and protection performance, and then an ultra-fast, high-sensitivity current differential protection method based on Bergeron model is proposed.

The rest of this paper is organized as follows: structure and intrinsic properties of HVDC transmission line are analyzed in section 2. Then, fundamental relations between line models and performance degradation of existing differential protections are 
revealed in section 3. Based on intrinsic properties of line in section 2, the accurate calculation method of 1-mode current, which is the prerequisite for ideal current differential protection, is also presented in section 3. In section 4, ultra-fast novel current differential protection scheme with high-sensitivity, including fault detection and identification of faulty pole, is developed, and theoretical analysis on the total response time of novel protection is also presented in this part. In section 5, the performance of the proposed current differential protection method is verified by simulation model of Wudongde $\pm 800 \mathrm{kV}$ HVDC project established in PSCAD/EMTDC. In the end, section 6 concludes this paper.

\section{HVDC transmission line and its intrinsic properties}

From the perspective of circuits, distributed capacitive current is the reason for mal-operation of differential protection. In fact, fast and accurate calculation of electrical quantities along transmission line by using measured signals at some points is the prerequisite for ultra-fast differential protection with high-sensitivity. Considering the calculation of electrical quantities with measured signals is closely related to transmission line parameters, structure and intrinsic properties of a HVDC transmission line is presented in this section, and its relationship with protection performance will be discussed in section 3 .

\subsection{Structure and decoupling of HVDC transmission line}

In HVDC system, overhead lines are widely used as power transmission carrier to connect different converters. Taking the overhead lines in $\pm 800 \mathrm{kV}$ HVDC system as an example, and its physical dimension is illustrated in Fig.1(a). The transmission line consists of three conducting paths, including positive pole, negative pole, and ground. Considering the two conducting transmission lines and ground are coupled with each other, detailed distributed parameter model of transmission line is illustrated in Fig.1(b).

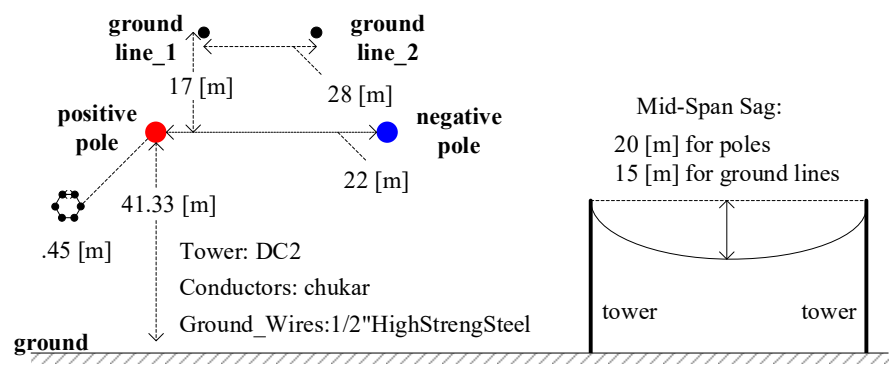

(a) The physical dimension of transmission lines

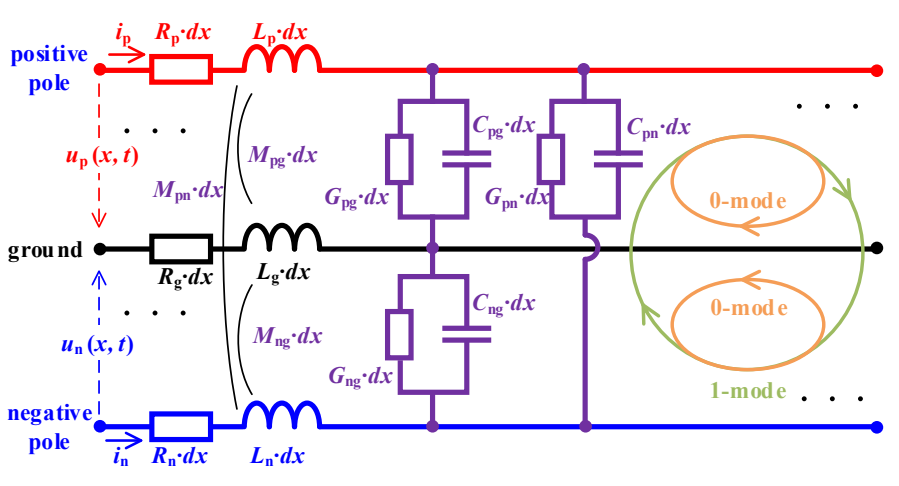

(b) The detailed distributed parameter model of transmission lines

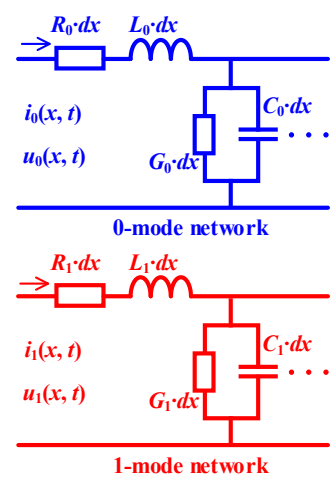

(c) The two decoupled mode networks.

Fig.1. Structure of the transmission line and its detailed distributed parameter model.

Referring to Fig.1(b), current and voltage on positive pole is described by (1). Current and voltage on negative pole can be expressed as the same form. It should be noted, the coupling effect between positive pole and negative pole is indicated by mutualinductance, shunt capacitance and shunt conductance, and some coefficients in (1) are variable. It means fast and accurate calculation of electrical quantities along transmission line by solving (1) directly is quite difficult. 


$$
\left\{\begin{array}{c}
\frac{\mathrm{d} u_{p}(x, t)}{d x}=-\left(\begin{array}{c}
R_{\mathrm{p}} i_{p}(x, t)+R_{g}\left[i_{p}(x, t)+i_{n}(x, t)\right]+\left(L_{\mathrm{p}}-M_{p g}\right) \frac{d i_{p}(x, t)}{d t} \\
-\left(M_{p g}-L_{g}\right) \frac{d\left[i_{p}(x, t)+i_{n}(x, t)\right]}{d t}+\left(M_{p n}-M_{n g}\right) \frac{d\left[i_{n}(x, t)\right]}{d t}
\end{array}\right) \\
\frac{\mathrm{d} i_{p}(x, t)}{d t}=-\left(\begin{array}{c}
G_{\mathrm{pg}} u_{p}(x, t)+G_{\mathrm{pn}}\left[u_{p}(x, t)-u_{n}(x, t)\right] \\
\left.+C_{p g} \frac{d u_{p}(x, t)}{d t}+C_{p n} \frac{d\left[\left(u_{p}(x, t)-u_{n}(x, t)\right]\right.}{d t}\right)
\end{array}\right)
\end{array}\right.
$$

To simplify the solving process, electrical quantities and transmission line parameters in (1) can be decoupled with Karenbauer conversion matrix [19]. The equivalent transmission line parameter in 0-mode and 1-mode network can be obtained by (2). Based on this conversion, the three loop circuits in Fig.1(b) are decoupled into 0-mode network and 1-mode network, just as illustrated in Fig.1(c) and (3). Consequently, with current and voltage in 0-mode network and 1-mode network decoupled, (3) can be solved separately and rapidly.

$$
\begin{aligned}
& R_{0}=R_{\mathrm{p}}+2 R_{\mathrm{g}} \quad R_{\mathrm{l}}=R_{\mathrm{p}} \\
& L_{0}=L_{\mathrm{p}}+2 L_{g}+M_{p n}-4 M_{p g} \quad L_{1}=L_{\mathrm{p}}-M_{p n} \\
& C_{0}=\frac{1}{2} C_{p g} \quad C_{1}=\frac{1}{2} C_{p g}+C_{p n} \\
& G_{0}=\frac{1}{2} G_{p g} \quad G_{1}=\frac{1}{2} G_{p g}+G_{p n} \\
& \left\{\begin{array}{l}
\frac{\mathrm{d} u_{i}(x, t)}{d x}=-\left(R_{i} i_{i}(x, t)+L_{i} \frac{d i_{i}(x, t)}{d t}\right) \\
\frac{\mathrm{d} i_{i}(x, t)}{d x}=-\left(G_{i} u_{i}(x, t)+C_{i} \frac{d u_{i}(x, t)}{d t}\right) \quad i=0,1
\end{array}\right.
\end{aligned}
$$

\subsection{Intrinsic properties of HVDC transmission line}

To solve (2) (3) quickly and accurately, characteristics of coefficients in (2) (3), which are actually decided by dimensions of transmission line, are studied in this part. Considering that electrical quantities with multiple frequency components propagate along line under many circumstances [20,21], relations between transmission line parameters in mode networks and frequency, including $\left(R_{0}, L_{0}, C_{0}, G_{0}\right)$ and $\left(R_{1}, L_{1}, C_{1}, G_{1}\right)$, are revealed in Fig.2.

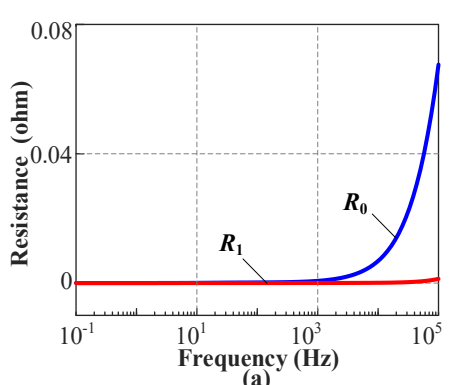

(a)

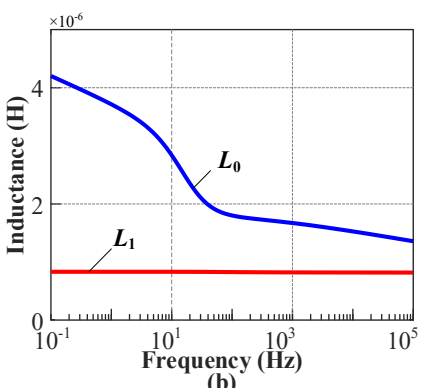

(b)

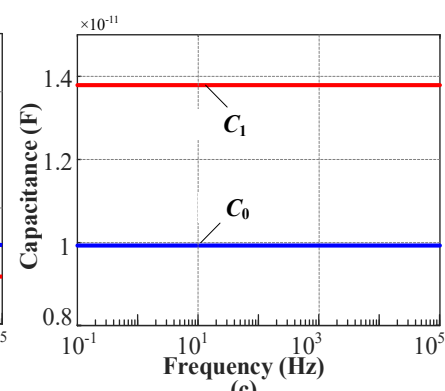

(c)

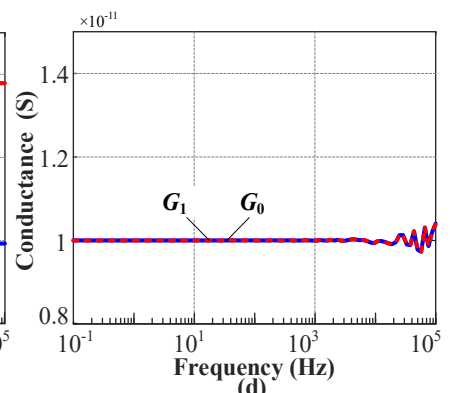

(d)

Fig.2. The frequency-dependent characteristics of parameters in mode networks of transmission lines.

(a) mode serial resistance; (b) mode serial inductance; (c) mode shunt capacitance; (d) mode shunt conductance.

When conducting current with different frequency, caused by eddy currents induced by changing magnetic field in the conductor itself, the real conducting area reduces with frequency, and the higher the frequency is, the smaller the real conducting area is. Considering that physical cross-section of ground is much larger than poles made of six- splitting leads, with skin depth larger than equivalent radius of poles, only skin effect of ground is apparent in low-frequency stage. In high-frequency stage, with skin depth getting smaller and comparable with equivalent radius of poles, skin effect of poles becomes apparent [22,23]. As a result, $R_{\mathrm{g}}$ increases with frequency obviously in low-frequency stage and $R_{\mathrm{p}}$ increases with frequency in high-frequency stage. 
Referring to (2), $R_{0}$ is equal to $R_{\mathrm{p}}+2 R_{\mathrm{g}}$ and $R_{1}$ is equal to $R_{\mathrm{p}}$. Therefore, $R_{0}$ increases with frequency during full-frequency stages, but $R_{1}$ is almost constant during low-frequency stage, as shown in Fig.2(a).

Similarly, because the physical dimension of ground is quite large, and its skin effect has become apparent during in lowfrequency stage, which affects the magnetic flux distribution and results in the self-inductance and mutual-inductance related to ground vary with frequency in low-frequency stage more obviously than high-frequency stage. Differently, because of the limited physical dimensions of poles, self-inductance and mutual inductance only related to poles, almost do not vary with frequency. As a result, $L_{0}$ decreases with frequency significantly during low-frequency stage but $L_{1}$ is almost constant during low and high frequency stages (referring to (2)), as shown in Fig.2(b).

Because capacitance and conductance are almost irrelevant with current distribution in ground and poles, $C_{0}, C_{1}, G_{0}$ and $G_{1}$ are almost constant during both low and high frequency stages, as shown in Fig.2(c) and Fig.2(d).

In conclusion, parameters of 0 -mode network vary with frequency during both low-frequency and high frequency stages. However, parameters in 1-mode network are almost constant during the low-frequency stage $(<1 \mathrm{kHz})$. It should be noted, the boundary frequency is decided by the dimension of transmission lines and its exposed environment, it could be obtained by field measurements or accurate mathematical model of transmission lines.

\section{Ideal current differential protection and its Prerequisite}

As mentioned before, relations between line model and protection performances have never be discussed thoroughly. In this part, the working principle of ideal current differential protection is presented first. Then, relations between line models and protection performances and the theoretical mechanism are revealed.

\subsection{Ideal current differential protection and its working principle}

Regardless of grounding modes of HVDC system, four differential protection units are configured at both terminals of positive pole and negative pole (pj, pk, nj, nk), just as illustrated in Fig.3. Voltages and currents at these four points are measured and indicated by $\left(u_{\mathrm{pj}}, i_{\mathrm{pj}}, u_{\mathrm{nj}}, i_{\mathrm{nj}}\right)$ and ( $\left.u_{\mathrm{pk}}, i_{\mathrm{pk}}, u_{\mathrm{nk}}, i_{\mathrm{nk}}\right)$. Typical internal faults include pole-to-pole short circuit faults (P2P), positive pole to ground faults $(\mathrm{P} 2 \mathrm{G})$, negative pole to ground faults $(\mathrm{N} 2 \mathrm{G})$, just as shown in Fig.3. The goal of differential protections is to distinguish internal faults from other working conditions by using the measured signals. Furthermore, P2P, P2G, and N2G should also be distinguished from each other so that relative DC circuit breakers are triggered to isolate the faulty area.

For working conditions except internal faults, referring to detailed distributed parameters line model in Fig.1(b), transmission line has no breakpoint and (1) is always satisfied along this line. Under this circumstance, there are two methods to calculate electrical quantities at any point $(\boldsymbol{r})$ on this line. One is to solve (1) by using measured signals at $\boldsymbol{j}$-point as initial values, and the other is to solve (1) by using measured signals at $\boldsymbol{k}$-point as initial values. The calculated electrical quantities of are indicated by

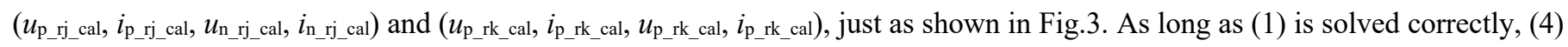
is always satisfied.

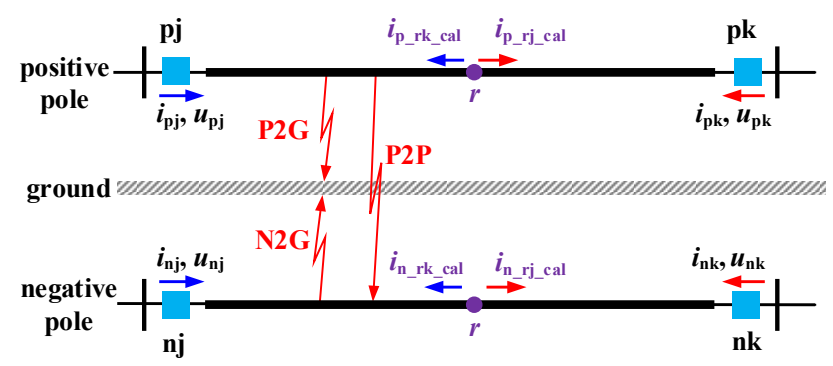

Fig.3. Configuration of differential protection for transmission line. 


$$
\begin{array}{ll}
u_{p_{-} r j_{-} c a l}(r, t)=u_{p_{-} r k_{-} c a l}(r, t) & i_{p_{-} r r_{-} c a l}(r, t)=-i_{p_{\beta_{1}} r k_{-} c a l}(r, t) \\
u_{n_{-} r j_{-} c a l}(r, t)=u_{n_{-} r k_{-} c a l}(r, t) & i_{n_{-} r j_{-} c a l}(r, t)=-i_{n_{-} r k_{-} c a l}(r, t)
\end{array}
$$

For internal faults, the fault point is actually a breakpoint on this line, resulting in (1) is not always satisfied along this line. Therefore, even if (1) are solved correctly, (4) are no longer satisfied.

Based on this difference during internal faults and other working conditions, ideal current differential protection is constructed in (5). As long as (1) could be solved correctly, $i_{\mathrm{p}_{-} \text {diff }}$ and $i_{\mathrm{n}_{\text {_liff }}}$ under internal faults are not zero, and for other working conditions, both $i_{\text {p_diff }}$ and $i_{\text {n_diff }}$ are equal to zero.

$$
\begin{aligned}
& i_{\mathrm{p}_{\mathrm{d}} \mathrm{diff}}=\left|i_{p_{-} r j_{\_} c a l}(r, t)+i_{p_{-} r k_{-} \text {cal }}(r, t)\right| \geq K_{\text {rel }} \cdot I_{\text {set }} \\
& i_{n_{-} \text {diff }}=\left|i_{n_{-} r j_{-} c a l}(r, t)+i_{n_{-} r k_{-} \text {cal }}(r, t)\right| \geq K_{\text {rel }} \cdot I_{\text {set }}
\end{aligned}
$$

\subsection{Relation between line models and ideal current differential protection}

As analyzed above, the prerequisite for ideal current differential protection is to calculate the differential current quickly and accurately. In existing current differential protection, different line models are used to solve (1), but not all of the line models can balance calculation accuracy and calculation speed, resulting in performance degradation of differential protections. Three typical line models are analyzed as follows:

\section{(1) Without using distributed parameters line model}

Without consideration of wave propagation process of electrical quantities along the line, the electrical quantities are assumed to be the functions of time $(t)$ only and not varying with distance $(x)$. Based on this assumption, (5) changes to (6), and ideal current differential protection degrades to the conventional current differential protection. As analyzed in section 2 , the wave propagation process along line is intrinsic and assumptions above are not valid during transient processes, resulting in maloperation of conventional current differential protection during initial transient stages under system disturbances and external faults are inevitable $[4,9]$.

$$
\begin{aligned}
& i_{\mathrm{p}_{-} \mathrm{d} i f f}=\left|i_{p_{-} r j_{-} c a l}(r, t)+i_{p_{-} r k_{-} c a l}(r, t)\right|=\left|i_{\mathrm{pj}}(r, t)+i_{p k}(r, t)\right| \geq K_{r e l} \cdot I_{s e t} \\
& i_{\mathrm{n}_{\text {ndiff }}}=\left|i_{n_{-} r j_{-} c a l}(r, t)+i_{n_{-} r k_{-} c a l}(r, t)\right|=\left|i_{n j}(r, t)+i_{n k}(r, t)\right| \geq K_{r e l} \cdot I_{\text {set }}
\end{aligned}
$$

\section{(2) Using frequency-dependent line model}

As presented in Section 2, the coefficients in equation (1), such as resistance and inductance relative to ground, vary with frequency significantly. Considering all working conditions, electrical quantities with multiple frequencies might distribute along transmission line, (1) could only be solved correctly in frequency domain using frequency-dependent line models which adopt parameters varying with frequency $[5,6,17]$. But the complex solving processes and the necessary sampling window result in a low calculation speed, and the response time of protection is prolonged to be $10 \sim 20 \mathrm{~ms}$.

\section{(3) Using Bergeron line model}

Another method to solve (1) is to decouple the three loop circuits into two mode networks first, and then solve (3). When assuming that parameters in mode networks are constant and serial resistance and shunt conductance could be neglected, frequencydependent line model degrades to Bergeron line model, and (3) changes to (7).

$$
\left\{\begin{array}{l}
\frac{\mathrm{d} u_{i}(x, t)}{d x}=-L_{i} \frac{d i_{i}(x, t)}{d t} \\
\frac{\mathrm{d} i_{i}(x, t)}{d x}=-C_{i} \frac{d u_{i}(x, t)}{d t} \quad i=0,1
\end{array}\right.
$$


With measured electrical quantities at $\boldsymbol{j}$-point or $\boldsymbol{k}$-point as initial values, by solving (7), electrical quantities in 0 -mode and 1-mode networks at any point $(\boldsymbol{r})$ along this line could be calculated easily [15]. However, assumptions in Bergeron line model make theoretical errors of calculation inevitable. Firstly, resistances cannot be neglected. Secondly, as discussed in section 2, resistances and inductances in mode networks are variable with frequency, resulting in the traveling wave dispersion. Therefore, when using Bergeron line model, although the calculation is fast, it is not accurate enough.

\subsection{Fast and accurate calculation of electrical quantities in 1-mode network}

Referring to Fig.2, transmission line serial inductance and shunt capacitance in 1-mode network are almost constant during both low-frequency stage and high-frequency stage. Therefore, the propagation speed $\left(v_{1}\right)$ and the wave impedance $\left(Z_{1}\right)$ in 1-mode network, decided by serial inductance and shunt capacitance, could be regarded as constant in the whole frequency band, just as illustrated in Fig.4. For comparison, the propagation speed $\left(v_{0}\right)$ and the wave impedance $\left(Z_{0}\right)$ in 0 -mode network are also illustrated. In other words, the calculation error of 1-mode electrical quantities, caused by the assumption that parameters are constant, could be eliminated, but the calculation error of 0 -mode electrical quantities, caused by the assumption that parameters are constant, is inevitable. Exact for this reason, only 1-mode differential current is suitable for fault detection.

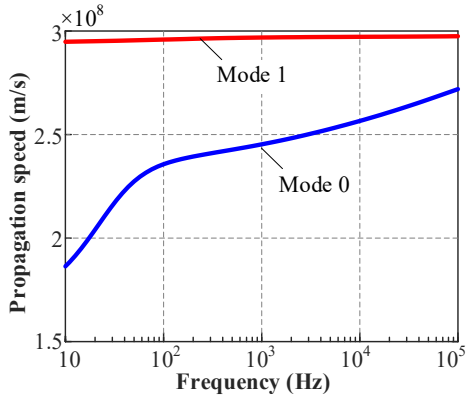

(a)

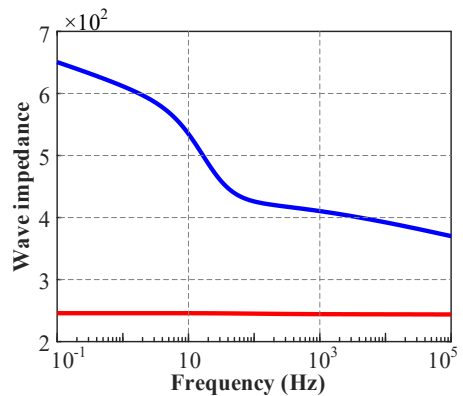

(b)

Fig.4. Propagation speed and wave impedance in mode networks.

(a) propagation speed of electrical quantities in mode networks; (b) wave impedance of electrical quantities in mode networks.

Another calculation error is that the transmission line is assumed lossless and the transmission line resistance and conductance are neglected in Bergeron model. To reduce even eliminate this error, the transmission line can be equivalent to several segments with a lumped resistor located at each end. Generally, the equivalent scheme of two segments with a resistance of 1/4.(R1.x) located at each end is sufficient for calculation accuracy. $R_{1}$ is the serial resistance of transmission line in 1-mode network, and it is almost constant during the low-frequency stage (referring to Fig.2). $x$ is the length of transmission line from $\boldsymbol{r}$-point to $\boldsymbol{k}$-point or $\boldsymbol{j}$-point. Under this circumstance, the 1-mode current of $\boldsymbol{r}$-point can be calculated with (8) and (9), and calculation errors, caused by assuming parameters are constant and resistance are negligible, could almost be eliminated.

$$
\begin{aligned}
& i_{1_{-} r_{-} c a l}\left(t-\tau_{1_{-j} j}\right)=\left(Z_{1}+R_{1} x_{r j} / 4\right)\left[u_{1_{-j} j}(t)-i_{1_{-j} j}(t)\left(Z_{1}+R_{1} x_{r j} / 4\right)\right] / 2 Z_{1}^{2} \\
& -R_{1} l \cdot\left[u_{1_{-} j}\left(t-\tau_{1_{-} r j}\right)-i_{1_{-} j}\left(t-\tau_{1_{-} r j}\right)\left(R_{1} x_{r j} / 4\right)\right] / 4 Z_{1}^{2} \\
& -\left(Z_{1}-R_{1} x_{r j} / 4\right)\left[u_{1_{-j} j}\left(t-2 \tau_{1_{-} r j}\right)+i_{1_{-} j}\left(t-2 \tau_{1_{-} j j}\right)\left(Z_{1}-R_{1} x_{r j} / 4\right)\right] / 2 Z_{1}^{2} \\
& i_{1 \_r k \_c a l}\left(t-\tau_{1 \_r k}\right)=\left(Z_{1}+R_{1} x_{r k} / 4\right)\left[u_{1 \_k}(t)-i_{1 \_k}(t)\left(Z_{1}+R_{1} x_{r k k} / 4\right)\right] / 2 Z_{1}^{2} \\
& -R_{1} x_{r k} \cdot\left[u_{1 \_k}\left(t-\tau_{1 \_r k}\right)-i_{1 \_k}\left(t-\tau_{1 \_r k}\right)\left(R_{1} x_{r k} / 4\right)\right] / 4 Z_{1}^{2} \\
& -\left(Z_{1}-R_{1} x_{r k} / 4\right)\left[u_{1 \_k}\left(t-2 \tau_{1 \_r k}\right)+i_{1 \_k}\left(t-2 \tau_{1 \_r k}\right)\left(Z_{1}-R_{1} x_{r k} / 4\right)\right] / 2 Z_{1}^{2}
\end{aligned}
$$

It should be noted, for any point (r) along line, all variants in (8) and (9) are known in advance and only simple computation, such as addition and subtraction, are need online. By using (8) and (9) to calculate low-frequency components of electrical quantities in 1-mode network, both calculation speed and accuracy could be ensured. 
Clearly, when using variants in 0-mode network, (8) and (9) can also be used to calculate 0-mode current at $\boldsymbol{r}$-point. But because $v_{0}, Z_{0}$ and $R_{0}$ vary significantly during full frequency band, the calculated 0 -mode electrical quantities deviate from real electrical quantities a lot. This is proved by the simulation in Section 5.

\section{Novel ultra-fast Current Differential Protection scheme with high-sensitivity}

Analysis in section 3 has shown that 1-mode current can be accurately and quickly calculated based on Bergeron model. In this section, a novel current differential protection scheme based on Bergeron line model is proposed and its performances is also discussed.

\subsection{Novel current differential protection scheme}

As mentioned before, functions of current differential protection scheme are to detect internal faults and to further distinguish P2P, P2G and N2G from each other. Therefore, the novel current differential protection scheme consists of the following two parts:

\section{(1) Internal fault detection based on 1-mode differential current}

Referring to (8) and (9), under all working conditions, low-frequency components of 1-mode current at $\boldsymbol{r}$-point, calculated by using measured signals at $\boldsymbol{j}$-point or $\boldsymbol{k}$-point, are indicated by $i_{1_{-} \text {rj_cal }}$ or $i_{1}$ _k__cal. If there is no fault point between $\boldsymbol{j}$-point and $\boldsymbol{r}$-point, $i_{1} \_$rj_cal is equal to the real current at $\boldsymbol{r}$-point. Similarly, if there is no fault point between $\boldsymbol{r}$-point and $\boldsymbol{k}$-point, $i_{1}$ _rk_cal is also equal to the real current at $\boldsymbol{r}$-point. It should be noted, $i_{1}$ rk_cal and $i_{1}$ rk_cal are defined in opposite directions. In this case, without fault point between $\boldsymbol{j}$-point and $\boldsymbol{k}$-point, instantaneous values of $i_{1}$ rj_cal $_{\text {cal }}$ and $i_{1}$ _rk_cal at $\boldsymbol{r}$-point are equal to real low-frequency components of 1 -

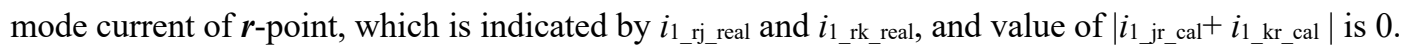

When an internal fault occurs, the fault point is located between $\boldsymbol{j}$-point and $\boldsymbol{r}$-point or between $\boldsymbol{r}$-point and $\boldsymbol{k}$-point. For the first case, Bergeron model is not satisfied between $\boldsymbol{j}$-point and $\boldsymbol{r}$-point, but still satisfied between $\boldsymbol{r}$-point and $\boldsymbol{k}$-point. As a result,

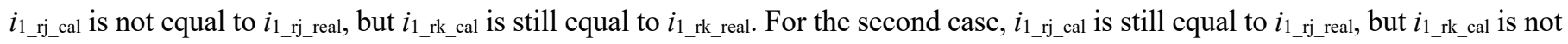
equal to $i_{1} \_$rk_real. In this case, value of $\mid i_{1}$ jr_cal $+i_{1} \_$kr_cal $\mid$is greater than 0 .

Based on the difference of $\mid i_{1}$ jr_cal $_{1}+i_{1}$ _kr_al $\mid$ between internal faults and other working conditions, such as steady state, system disturbances and external faults, the novel current differential protection criterion is constructed in (10).

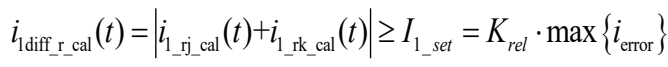

Where, $i_{1 \text { diff___cal }}$ is the calculated 1 -mode differential current. $K_{\text {rel }}$ is the reliability factor, and $i_{\text {error }}$ is the error of 1 -mode differential current caused by measurement. It should be noted, before calculating $(8) \sim(10)$, all high-frequency components of measured electrical quantities $(>1 \mathrm{kHz})$ have been filtered out first.

\section{(2) Faulty pole selection method based on mode quantities}

After an internal fault is detected, the false trip of protection on the healthy pole might occur if the faulty pole cannot be distinguished. To solve this problem, the faulty pole selection method based on mode quantities is proposed in this part.

Referring to Fig.3, the transmission line is divided into two segments by fault point $(\boldsymbol{f}$-point). Measured current at $\boldsymbol{j}$-point is actually feeding fault current from converters at $\boldsymbol{j}$-terminal and measured current at $\boldsymbol{k}$-point is actually feeding fault current from converters at $\boldsymbol{k}$-terminal. The real direction of fault current through poles under three typical faults, including P2G, P2P, and N2G, is illustrated in Fig.5. $i_{\mathrm{p}_{-} \mathrm{f}}$ is the fault current at $\boldsymbol{f}$-point through positive pole, and $i_{\mathrm{n}_{\mathrm{f}}}$ is the fault current at $\boldsymbol{f}$-point the through negative pole. Total fault current at $\boldsymbol{f}$-point includes two components from $\boldsymbol{j}$-point and $\boldsymbol{k}$-point, respectively, and it is indicated by $i_{\text {f. }}$ Defined positive directions of current through poles could be found in Fig.3. 


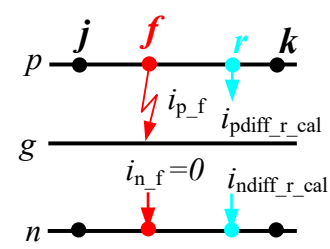

(a)

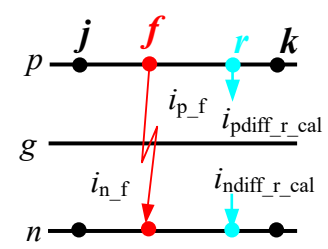

(b)

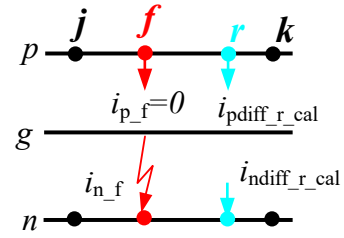

(c)

Fig.5. The real direction of fault current through poles under three typical faults.

(a) Positive pole to ground fault (P2G); (b) Pole-to-pole short circuit fault (P2P); (c) Negative pole to ground fault (N2G).

For $\boldsymbol{f}$-point on transmission line, the relationship between mode current and polar current could be described in (11), and currents through poles and in mode networks at $f$-point can be expressed in (12).

$$
\begin{aligned}
& {\left[\begin{array}{c}
i_{0_{0} \mathrm{f}}(t) \\
i_{1 \_\mathrm{f}}(t)
\end{array}\right]=\frac{\sqrt{2}}{2}\left[\begin{array}{l}
i_{\mathrm{p}_{-} \mathrm{f}}(t)+i_{\mathrm{n}_{\mathrm{f}}}(t) \\
i_{\mathrm{p}_{-} \mathrm{f}}(t)-i_{\mathrm{n}_{-} \mathrm{f}}(t)
\end{array}\right]} \\
& \int \mathrm{P} 2 \mathrm{G}: \quad\left[\begin{array}{l}
i_{\mathrm{p}_{\mathrm{f}}}(t) \\
i_{\mathrm{n} f \mathrm{f}}(t)
\end{array}\right]=\left[\begin{array}{l}
i_{\mathrm{f}}(t) \\
0
\end{array}\right], \quad\left[\begin{array}{l}
i_{0 \_\mathrm{f}}(t) \\
i_{1-\mathrm{f}}(t)
\end{array}\right]=\frac{1}{\sqrt{2}}\left[\begin{array}{l}
i_{\mathrm{f}}(t) \\
i_{\mathrm{f}}(t)
\end{array}\right] \\
& \left\{\mathrm{P} 2 \mathrm{P}: \quad\left[\begin{array}{c}
i_{\mathrm{p}_{\mathrm{f}}}(t) \\
i_{\mathrm{n}_{\mathrm{f}}}(t)
\end{array}\right]=\left[\begin{array}{c}
i_{\mathrm{f}}(t) \\
-i_{\mathrm{f}}(t)
\end{array}\right], \quad\left[\begin{array}{c}
i_{0_{0} \mathrm{f}}(t) \\
i_{1_{\mathrm{f}}}(t)
\end{array}\right]=\sqrt{2}\left[\begin{array}{c}
0 \\
i_{\mathrm{f}}(t)
\end{array}\right]\right. \\
& \mathrm{N} 2 \mathrm{G}:\left[\begin{array}{c}
i_{\mathrm{p}_{\mathrm{f}}}(t) \\
i_{\mathrm{n} f \mathrm{f}}(t)
\end{array}\right]=\left[\begin{array}{c}
0 \\
-i_{\mathrm{f}}(t)
\end{array}\right], \quad\left[\begin{array}{c}
i_{0 \mathrm{f}}(t) \\
i_{1 \mathrm{f}}(t)
\end{array}\right]=\frac{1}{\sqrt{2}}\left[\begin{array}{c}
i_{\mathrm{f}}(t) \\
-i_{\mathrm{f}}(t)
\end{array}\right]
\end{aligned}
$$

As shown in (12), an interesting phenomenon is found that for $\mathrm{P} 2 \mathrm{G}$ fault, 0 -mode current divided by 1 -mode current at $f$-point $\left(i_{0_{-}} \mathrm{f}(t) / i_{1_{-}} \mathrm{f}(t)\right)$ is 1 ; for $\mathrm{P} 2 \mathrm{P}$ fault, $i_{0_{-}} \mathrm{f}(t) / i_{1_{-}} \mathrm{f}(t)$ is 0 ; for $\mathrm{N} 2 \mathrm{G}$ fault, $i_{0_{-}} \mathrm{f}(t) / i_{1_{-}} \mathrm{f}(t)$ is -1 . Therefore, $i_{0} \mathrm{f}(t) / i_{1_{-}} \mathrm{f}(t)$ can be used to distinguish $\mathrm{P} 2 \mathrm{G}, \mathrm{P} 2 \mathrm{P}$ and $\mathrm{N} 2 \mathrm{G}$. However, $\boldsymbol{f}$-point is random on the transmission line and it is impossible to obtain $i_{0_{-}} \mathrm{f}(t) / i_{1_{-}} \mathrm{f}(t)$ online. To avoid calculate $i_{0} \mathrm{f}(t) / i_{1_{-}} \mathrm{f}(t)$ directly, one alternative way is proposed as follows:

In theory, calculated electrical quantities at $\boldsymbol{r}$-point along transmission line are obtained by solving equation (3) which assumes no breakpoint exists between $\boldsymbol{j}$-point and $\boldsymbol{k}$-point. Based on this assumption, by neglecting parameters vary with frequency, measured electrical quantities at $\boldsymbol{j}$-point propagate with the same speed and same wave impedance from $\boldsymbol{j}$-point to $\boldsymbol{f}$-point and $\boldsymbol{r}$ -

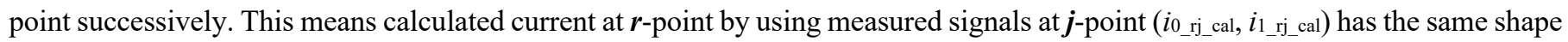
with feeding fault current at $\boldsymbol{f}$-point from $\boldsymbol{j}$-terminal, and a time difference exists. Similarly, calculated current at $\boldsymbol{r}$-point by using measured signals at $\boldsymbol{k}$-point $\left(i_{0}\right.$ _rj_cal, $\left.i_{1_{-} \text {rj_cal }}\right)$ has the same shape with feeding fault current at $\boldsymbol{f}$-point from $\boldsymbol{k}$-point, and a time difference exists. Referring to (8) (10) and taking time difference into consideration, calculated differential current at $\boldsymbol{r}$-point has the same shape with total fault current at $f$-point in mode networks. This means, when neglecting parameters vary with frequency,

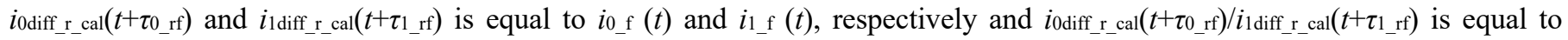
$i_{0 \_} \mathrm{f}(t) / i_{1_{-}} \mathrm{f}(t)$. It should be noted $\tau_{0_{-} \text {rf }}$ and $\tau_{1_{-} \mathrm{ff}}$ is decided by $v_{0}, v_{1}$ and distance between $\boldsymbol{r}$-point and $\boldsymbol{f}$-point $\left(x_{k f}\right)$, and they are variable with $x_{r f}$.

In fact, with $f$-point distributed randomly and $x_{k f}$ not known in advance, it is impossible to calculate $i_{0 \text { diff____cal }}\left(t+\tau_{0_{-} \text {rf }}\right) / i_{1 \text { diff_r_cal }}\left(t+\tau_{1_{-} \text {rf }}\right)$ online. For the convenience of calculation, $i_{0 \text { diff___cal }}(t) / i_{1 \text { diff___cal }}(t)$ is used to approximate $i_{0 \_}(t) / i_{1_{-} \mathrm{f}}$ $(t)$, and the faulty pole selection method based on mode quantities at $r$-point is constructed in (13).

$$
\begin{cases}\text { P2G: } & i_{\text {odiff___cal }}(t) / i_{\text {ldiff__cal }}(t)>S \\ \text { P2P: } & \left|i_{\text {odiff__cal }}(t) / i_{\text {diff__cal }}(t)\right|<S \\ \text { N2G : } & i_{\text {odiff___cal }}(t) / i_{\text {ddiff__cal }}(t)<-S\end{cases}
$$

$S$ is the threshold value, and it is used to compromise the inherent deviation between $i_{0 \text { diff____cal }}(t) / i_{1 \text { diff_r_cal }}(t)$ and $i_{0_{-}}(t) / i_{1} 1_{-}(t)$. Generally, $S$ with the value of 0.2 is enough to ensure reliability and sensitivity. 


\subsection{Analysis on total response time of novel differential protection scheme}

In the novel current differential protection, (10) is used to distinguish internal faults from other working conditions, and (13) is used to distinguish P2P, P2G, and N2G. With the measured signals obtained, only simple computations are needed, meaning calculation time is very small. However, the calculation time is only a very small part of the total response time of novel differential protection. Thus, the total response time is analyzed specially in this part, and the flowchart of this novel differential scheme is illustrated in Fig.6. As shown in Fig.6, the novel differential scheme consists of three steps:

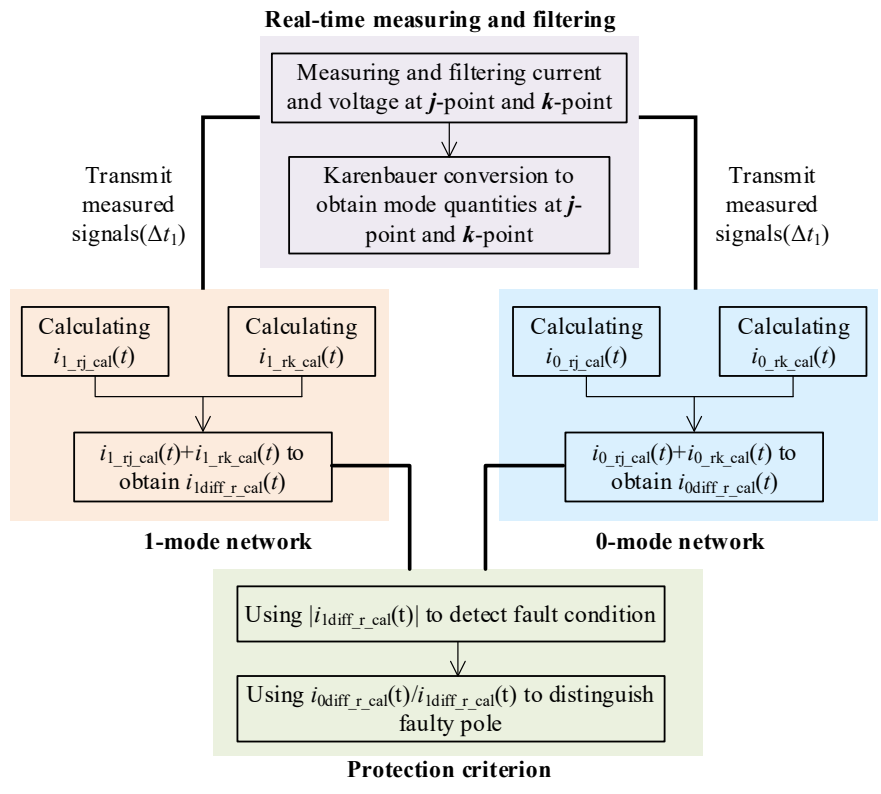

Fig.6. Flowchart of the novel differential protection.

The first step is real-time measuring and filtering of signals at $\boldsymbol{j}$-point and $\boldsymbol{r}$-point. In this step, signals are decoupled into mode quantities, and the signals obtained by sensors located at two ends of transmission line should be transmitted to the opposite end. The signal processing and transmitting result in a time delay of $\Delta t_{1}$, and it is decided by the length of transmission line between $\boldsymbol{j}$ point and $\boldsymbol{k}$-point and transmitting speed of signals. Taking the transmission line with a length of $800 \mathrm{~km}$ as an example, $\Delta t_{1}$ is about $\sim 3 \mathrm{~ms}$, and this time is inevitable for all kinds of differential protection.

The second step is using measured signals to calculate electrical quantities at $\boldsymbol{r}$-point, and it includes two parallel processes of calculating electrical quantities in 1-mode network and 0-network.

With $\boldsymbol{f}$-point distributed randomly along line, $\boldsymbol{r}$-point located anywhere between $\boldsymbol{j}$-point and $\boldsymbol{k}$-point, positions of these four points along line is illustrated in Fig.7. Distances between these points are indicated by $x_{\mathrm{rj}}, x_{\mathrm{rk}}, x_{\mathrm{fj}}$ and $x_{\mathrm{fk}}$, and length of transmission line is $l$.

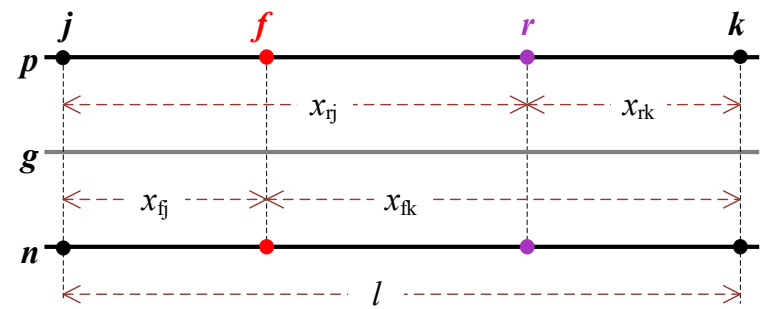

Fig.7. Locations of key points along transmission line, including $\boldsymbol{j}$-point, $\boldsymbol{f}$-point, $\boldsymbol{r}$-point and $\boldsymbol{k}$-point.

Referring to (8) and (9), the calculated current of $\boldsymbol{r}$-point at $\left(t-\tau_{1}{ }_{-} \mathrm{j}\right)$ is obtained by using measured signals of $\boldsymbol{j}$-point at $t$. Similarly, the calculated current of $\boldsymbol{r}$-point at $\left(t-\tau_{1_{-} \mathrm{rk}}\right)$ is obtained by using measured signals of $k$-point at $t$. To construct the current differential protection criterion in (10) and (13), the calculated current of $\boldsymbol{r}$-point by using measured signals at both sides should 
be consistent in time, meaning if $\tau_{1 \_ \text {rj }}$ is not equal to $\tau_{1_{-} \mathrm{rk}}$, additional delay of $\left|\left(x_{\mathrm{rj}}-x_{\mathrm{rk}}\right) / v_{1}\right|$ should be set. To avoid this additional delay, $\boldsymbol{r}$-point should be set at the middle of $\boldsymbol{j}$-point and $\boldsymbol{k}$-point, resulting in $\left|\left(x_{\mathrm{rj}}-x_{\mathrm{rk}}\right) / v_{1}\right|$ is 0 .

The third step is the calculation of protection criterion. In the novel differential protection, $i_{1}$ rij_cal and $i_{1}$ rk_cal at same time are used to calculate $i_{1 \text { diff___cal }}$ and distinguish internal faults from other working conditions. In this case, the measured signals in these two terminals at $t=t_{0}$ are assumed the traveling wave propagated from the reference point $l / 2 v_{1}$ ago. This assumption is valid for working conditions except internal faults. For internal faults, the time delay of fault detection based on (10) is defined as $\Delta t_{21}$, and it is decided by fault location and fault type. Detailed reason is analyzed as follows: with $\boldsymbol{r}$-point at the middle of transmission line and $\boldsymbol{f}$-point located randomly between $\boldsymbol{j}$-point and $\boldsymbol{k}$-point, the 1 -mode fault traveling wave will propagate to $\boldsymbol{j}$-point at $t=t_{0}+x_{\mathrm{fj}} / \nu_{1}$ after fault occurrence at $t=t_{0}$, meaning the fault current calculation time-delay of $\boldsymbol{j}$-point is $x_{\mathrm{fj}} / v_{1}$. Similarly, the 1 -mode fault traveling wave will propagate to $\boldsymbol{k}$-point at $t=t_{0}+x_{\mathrm{fk}} / v_{1}$ after fault occurrence at $t=t_{0}$, meaning the fault current calculation time-delay of $\boldsymbol{j}$-point is $x_{\mathrm{fk}} / v_{1}$. According to (8), (9) and (10), as long as measured signals at $\boldsymbol{j}$-point or $\boldsymbol{k}$-point consist of 1-mode fault current component, the calculated differential 1-mode current in (10) rises. Thus, for metallic fault with large fault current, the calculated 1-mode differential current will reach the threshold value once the fault traveling wave reached one of the $\boldsymbol{j}$-point and $\boldsymbol{k}$-point, and calculation time delay of (10) is the minimum of $\left(x_{\mathrm{fj}} / v_{1}, x_{\mathrm{fk}} / v_{1}\right)$. However, for high-resistance fault with small fault current, only if both measured signals at $\boldsymbol{j}$-point and $\boldsymbol{k}$-point consist of 1 -mode fault current component, the calculated 1-mode differential current in (10) will reach the threshold value, and the calculation time delay is the maximum of $\left(x_{\mathrm{fj}} / v_{1}, x_{\mathrm{fk}} / v_{1}\right)$. Therefore, regardless of fault transition impedance, with $\boldsymbol{r}$-point at the middle of transmission line and $\boldsymbol{f}$-point located randomly between $\boldsymbol{j}$-point and $\boldsymbol{k}$-point, the time delay of fault detection $\Delta t_{21}$ varies from 0 to $l / v_{1}$.

Similar to analysis in 1-mode network, the propagation delay of 0 -mode fault current traveling from $\boldsymbol{f}$-point to $\boldsymbol{j}$-point is $x_{\mathrm{fj}} / v_{0}$, and the time is $x_{\mathrm{fk}} / v_{0}$ for 0 -mode fault current traveling from $\boldsymbol{f}$-point to $\boldsymbol{k}$-point. It should be noted, the propagation speed of 0 -mode current is slower than 1-mode current, meaning the calculation time delay of 0 -mode differential current in (13) is larger than 1mode differential current in (10). Time delay of faulty pole selection after internal faults detection is defined as $\Delta t_{22}$. To ensure the reliability, the faulty pole selection can be performed after a delay of $\Delta t_{22}=\max \left(x_{\mathrm{fj}} / v_{0}-x_{\mathrm{fj}} / v_{1}\right)=\left(l / v_{0}-l / v_{1}\right)$ after internal fault detection.

Based on the analysis above, although calculation time based on Bergeron model is very small and could be neglected, but $\Delta t_{1}$ for signals processing and transmitting from one terminal to opposite terminal and $\Delta t_{2}=\Delta t_{21}+\Delta t_{22}$ for signals propagating along these key points, cannot be ignored. The total response time of novel current differential protection is $\Delta t_{1}+\Delta t_{2}$, and it varies with line length and specific fault location.

\section{Case studies}

According to theoretical analysis above, the novel current differential protection is closely related with electrical wave propagation process along transmission lines and it is suitable for HVDC system with any kind of converters. In addition, due to the accurate and rapid calculation of 1-mode current, mal-operation of novel current differential protection under working conditions except internal faults, e.g. large system disturbances and external faults, is reliably avoided, and its rapid and sensitive response to diversified internal faults is ensured. In this part, case studies are conducted to verify the excellent performance of the novel current differential protection.

\subsection{Simulation model of Wudongde LCC-VSC-HVDC project}

Without loss of generativity, simulation model of Wudongde HVDC project in China, which is a bipolar LCC-VSC-HVDC transmission system with three converters, is established in PSCAD/EMTDC. Diagram of the simulation model is illustrated in Fig.8. 


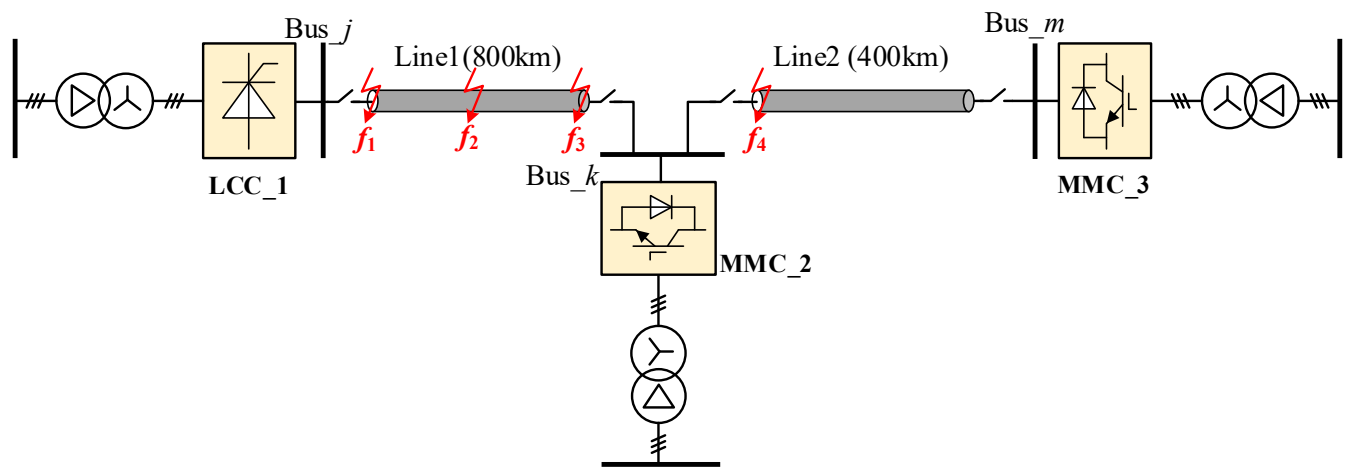

Fig.8. Diagram of Wudongde HVDC transmission system.

Table 1

The Converter Station Parameters

\begin{tabular}{lccc}
\hline \multicolumn{1}{c}{ Parameters } & LCC_1 & MMC_2 & MMC_3 \\
\hline Rated power/MW & 5000 & 3000 & 2000 \\
Rated dc voltage $/ \mathrm{kV}$ & \pm 800 & \pm 800 & \pm 800 \\
Rated ac voltage $/ \mathrm{kV}$ & 525 & 525 & 525 \\
Level number of each valve group of MMC & $\backslash$ & 207 & 207 \\
Pulse number of LCC & 12 & $\backslash$ & $\backslash$ \\
Transformer ratio & $525 / 172$ & $525 / 190$ & $525 / 189.5$ \\
Capacitance of SM/ $\mu \mathrm{F}$ & $\backslash$ & 18000 & 12000 \\
Reactance of bridge arm $/ \mathrm{mH}$ & 100 & 61.2 & 41.2 \\
Smoothing reactor $/ \mathrm{mH}$ & 150 & 75 & 75 \\
\hline
\end{tabular}

As shown in Fig.8, LCC_1 is a rectifier, and it works in constant voltage control mode; MMC_2 and MMC_3 are inverters and they work in constant power control mode. Power flows from LCC_1 to MMC_2 and MMC_3. By using MMC instead of LCC as inverters receiving power from DC system, no risk of commutation failures is avoided. Detailed parameters of three converters are listed in Table 1. The transmission line between these converters is Line1 and Line2, and detailed dimensions of these lines could be found in Fig.1(a).

\subsection{Discussion on simulation results}

\subsubsection{Performance of novel current differential protection under working conditions except internal faults}

As mentioned before, caused by the electric wave propagation during transient processes, mal-operation of conventional current differential protection could occur. However, by accurate calculation of differential current which takes the electric wave propagation processes into consideration, mal-operation of novel current differential protection can be avoided. In this part, working condition of large system disturbances and most severe external faults are discussed.

\section{(1) Simulation of large system disturbance}

To simulate working condition of large system disturbances, MMC_3 is controlled to be blocked at $t=3.5 \mathrm{~s}$ in the simulation, which could excite large transient process along with the power re-distribution in power system. The reason for conducting this case study is: mal-operation of conventional current differential protection could occur under this case but the novel differential protection has the capacity of resisting this transient process. Simulation results are illustrated in Fig.9.

As shown in Fig.9(a), with current approximate to be constant during steady state, the current varies with time $(t)$ and distance $(x)$ slightly, conventional polar differential current ( $i_{p_{-}}$diff), obtained by adding measured current of $\boldsymbol{j}$-point and $\boldsymbol{k}$-point at the same time directly, is almost zero in steady state before $t=3.5 \mathrm{~s}$. However, when MMC_ 3 is suddenly blocked at $t=3.5 \mathrm{~s}$, large system 
disturbance occurs, and with transient current consisting of multiple frequency components, the current varies with time $(t)$ and distance $(x)$ significantly. In this case, $i_{p_{-} \text {diff }}$ (conventional differential current) is quite large, and could exceed the threshold, resulting in mal-operation. In practical project, a time delay of hundreds of milliseconds is set to avoid this mal-operation. It should be noted, $\Delta t_{1}$ in Fig.9(a) is the time for measured signals transmitted from one terminal to opposite terminal, and it is inevitable for all kinds of differential protections.

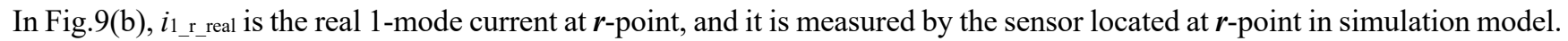
$i_{1 \_ \text {rij_cal }}$ is the calculated 1 -mode current at $\boldsymbol{r}$-point by using measured signals at $\boldsymbol{j}$-point (referring to (8)). Consistent with theoretical analysis in section 4 , without fault point between $\boldsymbol{j}$-point and $\boldsymbol{r}$-point, because $v_{1}, Z_{1}$ and $R_{1}$ are almost constant during lowfrequency stage, $i_{1}$ rij_cal has the same shape with $i_{1}$ r_real $_{\text {real }}$. It should be noted, referring to (8), because the calculated current at $t$ corresponds to the real current of $r$-point at $\left(t-\tau_{1}\right.$ rij $)$, a time difference of $\tau_{1-\text { rj }}$ exists between $i_{1_{-} \text {rj_cal }}$ and $i_{1}$ r $_{-}$real. What analyzed above

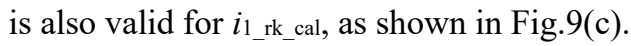

Because of the accurate calculation of 1-mode current based on Bergeron model, the calculated 1-mode currents at $\boldsymbol{r}$-point

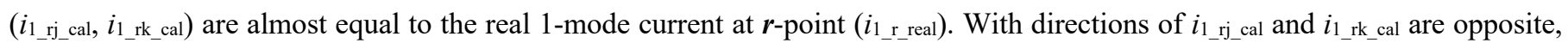
protection criterion in (10) is not satisfied and mal-operation of novel current differential protection does not occur, just as shown in Fig.9(d).

(a)

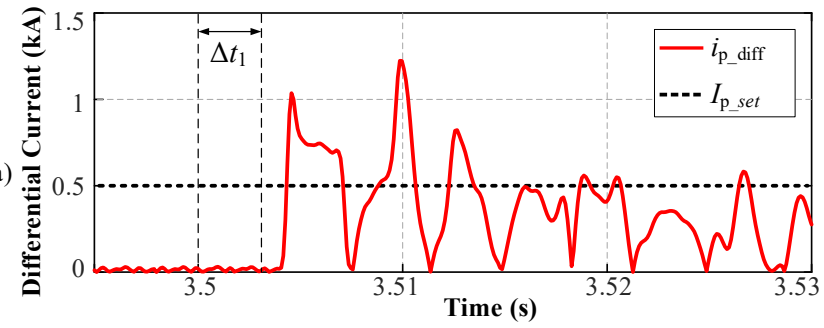

(b)
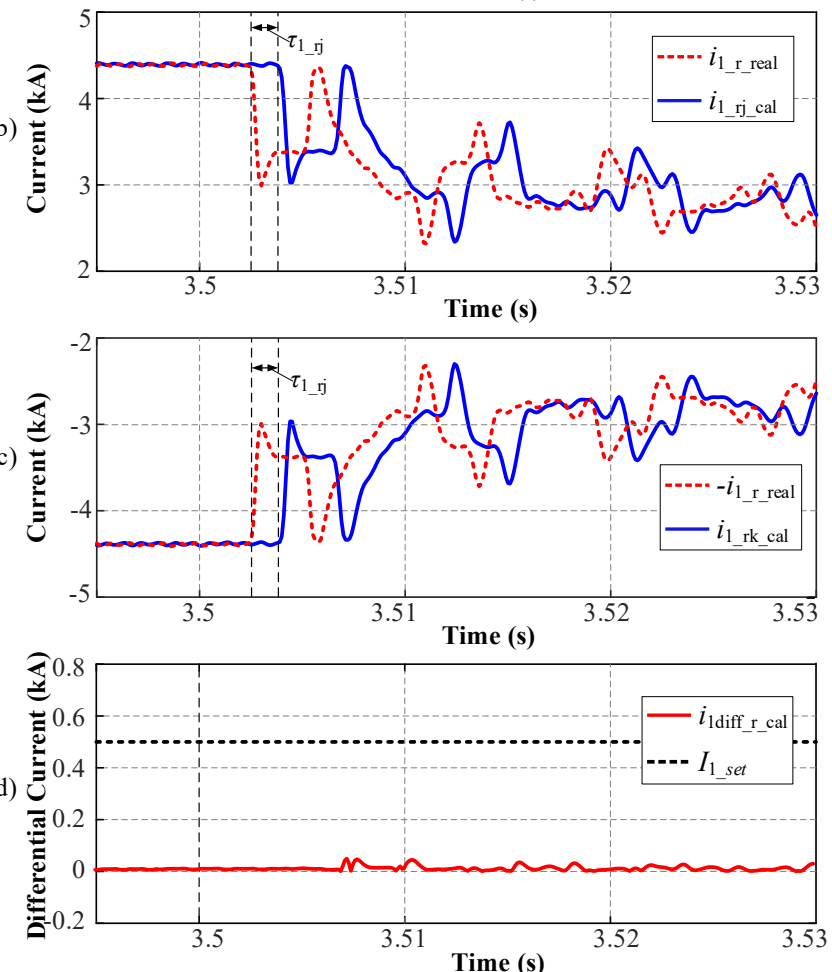

Fig.9. Simulation results of protections on Line1 when MMC_3 suddenly blocked.

(a) conventional polar differential current; (b) 1-mode calculated current with the measured quantities of $j$;

(c) 1-mode calculated current with the measurement quantities of $k$; (d) 1-mode differential current. 


\section{(2) Simulation of external fault}

External fault generating large-fault current is another typical working condition that is hard to distinguish from internal faults for protection. Therefore, case study on external nearby metallic P2P fault $\left(f_{4}\right.$ in Fig.8) is conducted in this part to verify the correct operation of novel current differential protection. In addition, by illustrating calculated current with or without low-pass filter, the necessity for low-pass filter is also discussed in this part. With metallic fault occurring at $3.5 \mathrm{~s}$, simulation results are illustrated in Fig.10.

Similar with the simulation results in Fig.9(a), the conventional polar differential current ( $i_{p_{-}}$diff) is almost zero before $3.5 \mathrm{~s}$, as shown in Fig.10 (a). However, transient differential current ( $\left.i_{\mathrm{p} \_ \text {diff }}\right)$ with large amplitude and long duration appears after the signal processing and transmitting delay $\left(\Delta t_{1}\right)$. Therefore, long-time delay of hundreds of milliseconds must be set to avoid this maloperation.

As shown in Fig.10(b), consistent with theoretical analysis in section 4, without fault point between $\boldsymbol{j}$-point and $\boldsymbol{r}$-point, based on the accurate calculation of 1 -mode current after low-pass filtering, $i_{1}$ rj_cal $_{-}$has the same shape with $i_{1} \mathrm{r}_{-}$real, and only a time difference exists.

To discuss the necessity for low-pass filter, the calculated differential current with filtering $\left(i_{1 \text { diff__ } \mathrm{r} \text { call }}\right)$ and without filtering ( $\left.i_{1 \text { diff___cal2 }}\right)$ are illustrated in Fig.10(c) respectively. As shown in Fig.10(c), $i_{1 \text { diff___cal2 }}$ reaches peak values periodically during transient stage and it could exceed the threshold, meaning mal-operation of current differential protection by using $i_{1 \text { diff___cal2 }}$ as criterion is possible. In fact, these periodical peaks are caused travelling wave dispersion and limited sampling frequency of $10 \mathrm{kHz}$, resulting in high-frequency component cannot be measured and calculated accurately. To suppress these periodical peaks, lowpass filter with cut-off frequency of $1 \mathrm{kHz}$ is used. As shown in Fig.10(c), consistent with the theoretical analysis in section 4, for working conditions without internal faults, $i_{\text {diff___cal }}$ is almost zero, and no mal-operation of novel current differential protection happens.

(a)

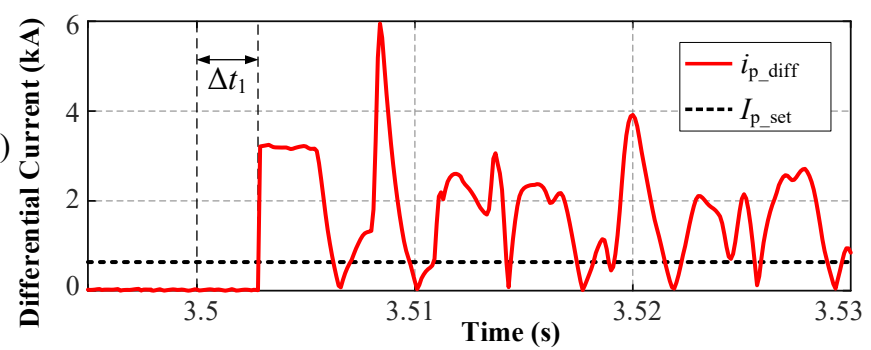

(b)
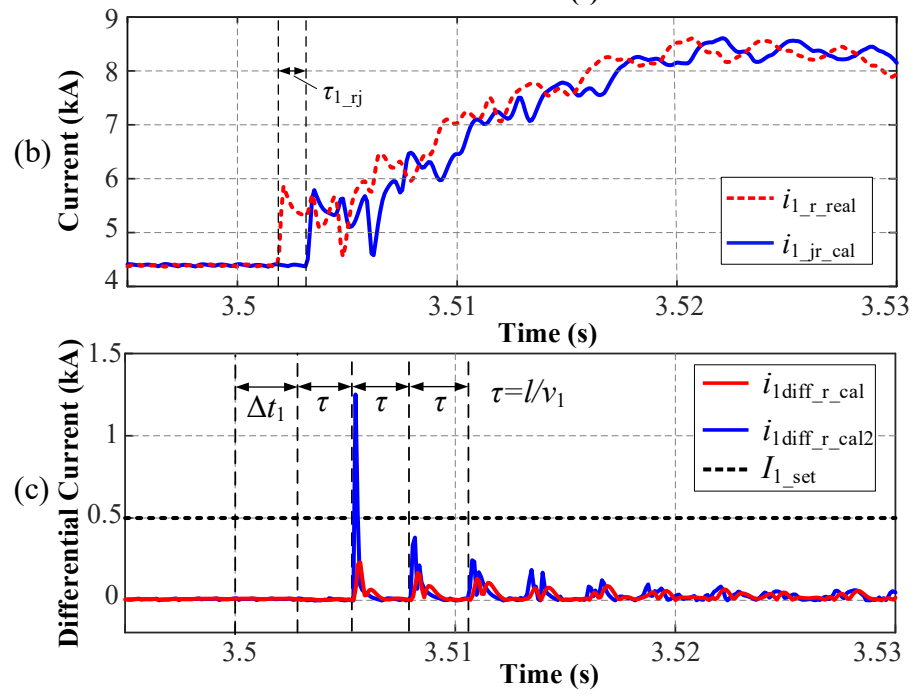

Fig.10. Simulation results of differential protections on Line1 when external metallic P2P occurs. 
(a) conventional polar differential current; (b) 1-mode current at $\boldsymbol{r}$-point; (c) 1-mode differential current.

\subsubsection{Performance of novel current differential protection under internal faults}

As analyzed before, regardless of fault type, fault location and fault transition resistance, the novel current differential protection could respond to any kind of internal faults within several milliseconds. However, the response time could be affected by these factors. To verify the theoretical analysis before, case studies of diversified internal faults are conducted in this part.

\section{(1) Internal metallic positive pole to ground fault (P2G) at the midpoint of Line1 $\left(f_{2}\right)$}

Scenario of P2G at midpoint of line occurring at 3.5s is conducted in this part, and simulation results are illustrated in Fig.11. Based on simulation results, protection performance, including fault detection time and faulty pole selection time, are analyzed. In addition, the calculation accuracy of 0 -mode current and 1-mode current are also discussed in this part.

In Fig.11(a), $i_{0 \text { fault_real }}$ is the real 0 -mode fault current at $\boldsymbol{f}$-point after filtering. $i_{\text {odiff_con }}$ is conventional 0 -mode differential current, and it is obtained by adding 0 -mode components of measured signals at $\boldsymbol{j}$-point and $\boldsymbol{r}$-point after filtering directly. $i_{0 \text { diff___cal }}$ is the 0-mode calculated differential current based on Bergeron model (using 0-mode parameters and electrical quantities measured at $\boldsymbol{j}$-point and $\boldsymbol{r}$-point after filtering in (8) (10)).

In Fig.11(b), $i_{1 \text { fault_real }}$ is the real 1-mode fault current at $f$-point after filtering. $i_{1 \text { diff_con }}$ is conventional 1-mode differential current, and it is obtained by adding 1 -mode components of measured signals at $\boldsymbol{j}$-point and $\boldsymbol{r}$-point after filtering directly. $i_{1 \text { diff___cal }}$ is 1-mode calculated differential current based on Bergeron model (referring to (8) (10)).

(a)

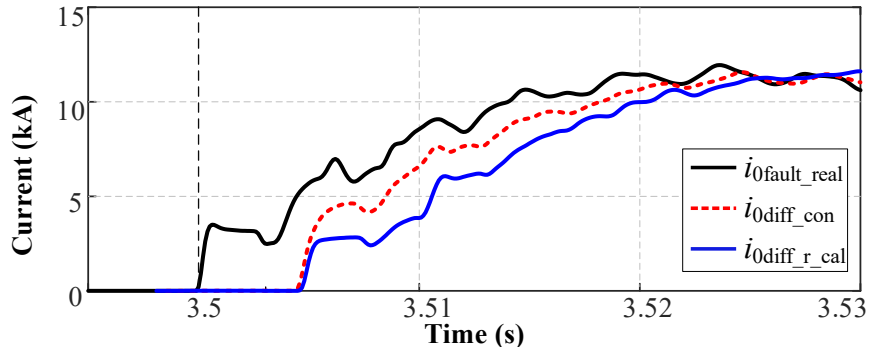

(b)
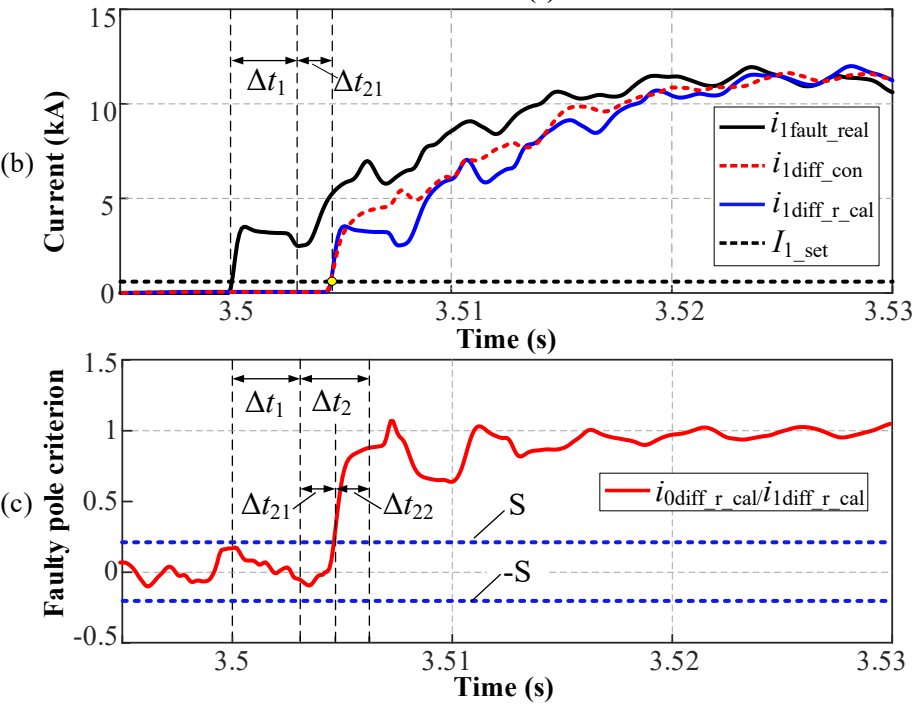

Fig.11. Mode differential current and results of protection under metallic $\mathrm{P} 2 \mathrm{G}$ fault at midpoint of Line $1\left(f_{2}\right)$.

(a) 0-mode current; (b) 1-mode current and fault detection result; (c) faulty pole selection result.

With $\boldsymbol{f}$-point coinciding with $\boldsymbol{r}$-point, as long as the calculation of mode current at $\boldsymbol{r}$-point by using measured signals at $\boldsymbol{j}$-point and $\boldsymbol{k}$-point is accurate, the calculated differential current is equal to the real fault current at $\boldsymbol{r}$-point. In Fig.11(a) and Fig.11(b), because $i_{0 \text { diff_con }}$ and $i_{1 \text { diff_con }}$ are obtained without considering the fact that current varies with $(t)$ and $(x)$ which could be described 
by wave propagation process, $i_{0 \text { diff_con }}$ and $i_{1 \text { diff_con }}$ have different shapes with $i_{0 \text { fault_real }}$ and $i_{1 \text { fault_real }}$ respectively. In the novel current differential protection, $i_{1 \text { diff_r_cal }}$ is almost the same with $i_{1 \text { diff_real }}$ but $i_{0 \text { diff___cal }}$ deviates from $i_{0 \text { diff_real }}$ significantly, meaning the calculation of 1-mode current is much more accurate than 0 -mode current. The results are consistent with analysis in section 4.1. Because $v_{1}, Z_{1}$ and $R_{1}$ are constant during low-frequency stage, 1-mode current maintains same shape during the propagation process. Therefore, $i_{1 \text { diff___cal }}$ can reflect real 1 -mode fault current at $\boldsymbol{f}$-point $\left(i_{1 \text { fault_real }}\right)$ by using Bergeron model to describe wave propagation process between key points, and it is used as fault detection criterion.

Another interesting phenomenon is, as shown in Fig.11(b), total response time for fault detection by using $i_{1 \text { diff_r_cal }}$ is $\Delta t_{1}+\Delta t_{21}$. Consistent with analysis in section $4.2, \Delta t_{1}$ is for signal processing and transmitting, and it is inevitable for any kind of differential protections $(\sim 3 \mathrm{~ms}$ for line with length of $800 \mathrm{~km}) . \Delta t_{21}$ is caused by electrical quantities propagating along key points, and it is $l /\left(2 v_{1}\right)$ here.

Faulty pole selection result of novel differential protection scheme is illustrated in Fig.11(c). In Fig.11(c), $i_{0 \text { diff_r_cal }}(t) / i_{1}$ diff_r_cal $(t)$ is used for faulty pole selection, and $S$ of 0.2 is designed as setting values to avoid false trip of protections on heathy poles. Consistent with analysis in 4.1 and 4.2 , because $v_{0}$ is smaller than $v_{1}$, a time delay of $\Delta t_{22}\left(l / v_{0}-l / v_{1}\right)$ for 0 -mode current propagation is set in faulty pole selection after fault detection. Considering $\Delta t_{1}, \Delta t_{21}$ and $\Delta t_{22}$, the fault can be detected in $4.35 \mathrm{~ms}$ and the faulty pole can be selected in $6.04 \mathrm{~ms}$.

\section{(2) Internal nearby pole-to-pole short circuit fault (P2P) at terminal $j\left(f_{1}\right)$}

Scenario of internal nearby $\mathrm{P} 2 \mathrm{P}$ at $f_{1}$ occurring at $3.5 \mathrm{~s}$ is conducted in this part, and simulation results are illustrated in Fig.12. In the case of pole-to-pole short circuit fault, the fault current only flows through the positive line and the negative line. Therefore, the 0 -mode component of the real fault current $i_{0 \text { fault }}$ real is 0 , and both the calculated 0 -mode component of the fault current $i_{0 \text { diff___cal }}$ and the conventional 0 -mode differential current $i_{0 \text { diff_con }}$ are very small, as shown in Fig.12(a). This is another reason why 0-mode current cannot be used for fault detection.

In Fig.12(b), the shapes of $i_{1 \text { diff___cal }}$ and $i_{1 \text { fault_real }}$ are no longer the same. The reason is $\boldsymbol{f}$-point does not coincide with $\boldsymbol{r}$-point, and this could be explained by analysis in Section 4.1. When calculating $i_{1 \text { diff_r_cal }}$ using Bergeron model, it is assumed that measured signals at $\boldsymbol{j}$-point contain the traveling wave propagated from $\boldsymbol{r}$-point and measured signals at $\boldsymbol{k}$-point also contain the traveling wave propagated from $\boldsymbol{r}$-point. However, when the $\boldsymbol{f}$-point is inconsistent with $\boldsymbol{r}$-point, the time parameters used in (8) and (9) are different with the real values. Thus, calculation errors will inevitably occur.

In Fig.12(b), the fault can be detected once the fault signals are received, meaning $\Delta t_{21}$ is quite small. This is because the fault signal is measured at $\boldsymbol{j}$-point immediately after the fault occurrence. Therefore, the calculated differential current rises immediately after $\Delta t_{1}$, and the fault is identified as internal fault. This is consistent with the analysis in Section 4.2. So, the shortest fault detection time will be only the sum of the signal transmitting time and signal processing time. In this case, the fault is detected in $3.01 \mathrm{~ms}$.

The faulty pole selection result is shown in Fig.12(c). Since $i_{0 \text { diff_r_cal }}$ is almost zero before and after the fault occurrence, and $i_{1 \text { diff_r_cal }}$ rises once the fault occurs, the faulty pole could be easily and reliably selected in $4.70 \mathrm{~ms}$ based on (13). 
(a)

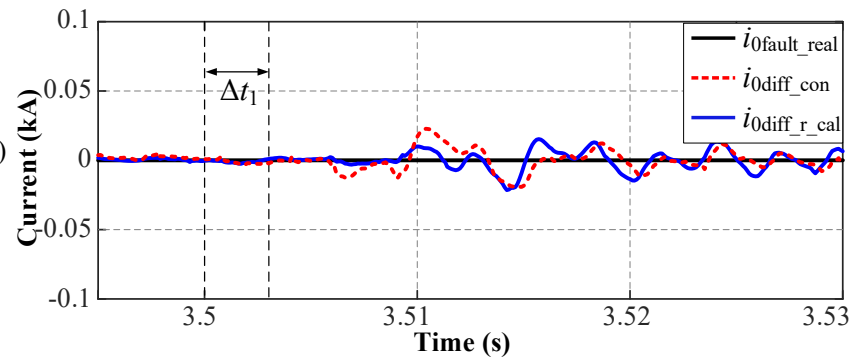

(b)
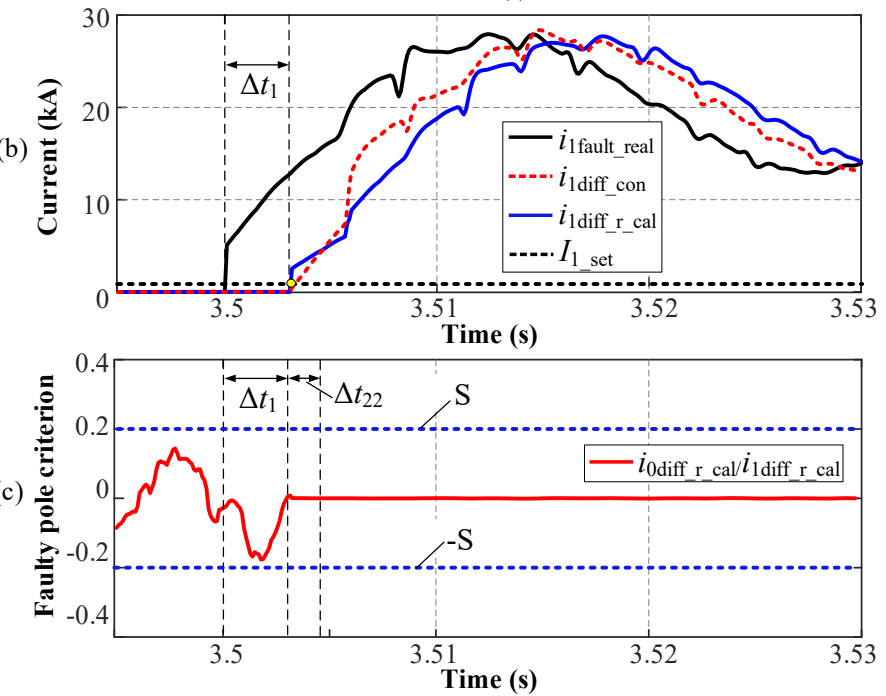

Fig.12 Mode differential current and results of protection under nearby metallic P2P fault of Line1 $\left(f_{1}\right)$

(a) 0-mode current; (b) 1-mode current and fault detection results; (c) faulty pole selection results.

\section{(3) Internal nearby negative pole to ground fault (N2G) at terminal $k\left(f_{3}\right)$}

To prove sensitivity of the novel current differential protection method under faults with small-current is better than existing current differential protection method, scenario of internal nearby negative to ground fault $(\mathrm{N} 2 \mathrm{G})$ with large transition resistance of $500 \Omega$ occurring at $\mathrm{t}=3.5 \mathrm{~s}$ is conducted, and simulation results are shown in Fig. 13 .

In Fig.13(a) and Fig.13(b), because $\boldsymbol{f}$-point is inconsistent with $\boldsymbol{r}$-point, the shapes of $i_{0 \text { diff___cal }}$ and $i_{0 \text { fault_real }}$ are different, and so are $i_{1 \text { diff___cal }}$ and $i_{1 \text { fault_real. }}$. Consistent with the theoretical analysis in Section 4.2 , both $i_{0 \text { diff___cal }}$ and $i_{1 \text { diff___cal }}$ rise immediately after the signal processing and transmitting delay. However, due to the large fault transition resistance, $i_{1 \text { diff___cal }}$ can reach the protection threshold only after the 1-mode fault traveling wave propagated from the fault point to both $\boldsymbol{k}$-point and $\boldsymbol{j}$-point, as shown in Fig.13(b). So, the time delay of fault detection is the longest and $\Delta t_{21}=l / v_{1}$. In this case, the fault detection time is $5.65 \mathrm{~ms}$, and the faulty pole selection time is $7.34 \mathrm{~ms}$, as shown in Fig.13(c). Therefore, the novel current differential protection has a good performance of sensitivity and can reliably detect the fault with high transition resistance. Considering $\Delta t_{1}, \Delta t_{21}$ and $\Delta t_{22}$, the total response time of novel differential protection scheme is decided by line length, specific fault location and fault transition resistance. For line with length of $800 \mathrm{~km}$, the possible longest response time is less than $7.5 \mathrm{~ms}$, which is much shorter than the response time of existing differential protections. 
(a)
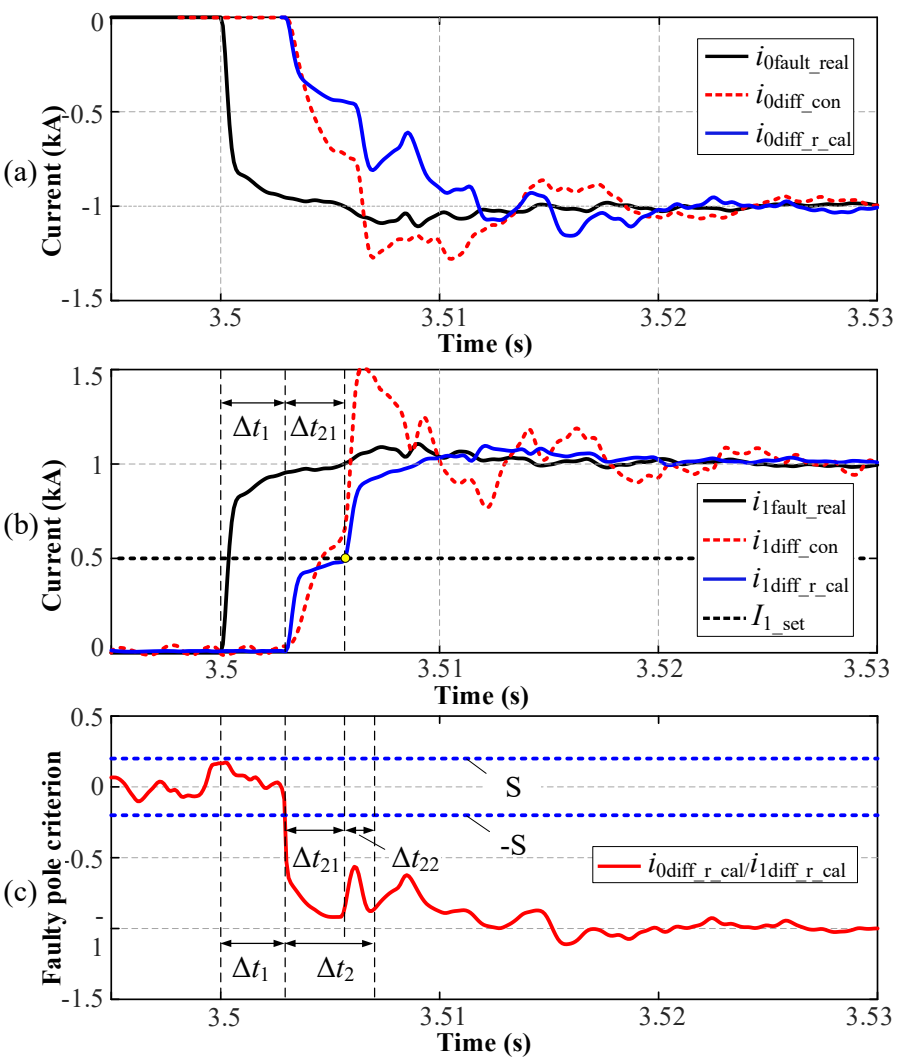

Fig.13. Mode current and results of protection under nearby high-resistance N2G fault of Line1 $\left(f_{3}\right)$.

(a) 0-mode current; (b) 1-mode current and fault detection results; (c) faulty pole selection results.

\subsubsection{Simulation results of other working conditions}

Simulation on many other scenarios, including diversified internal faults, some non-ideal factors, e.g. signal noise and line parameter errors, are also conducted. However, due to limited space, only important performance indicators of the novel current differential protection, including fault detection time and faulty pole selection time are presented in Table 2 .

Table 2

Fault detection and faulty pole selection results

\begin{tabular}{|c|c|c|c|c|}
\hline Fault type & $\begin{array}{c}\text { Distance from } j \\
\text { to fault point }\end{array}$ & $\begin{array}{l}\text { Transition } \\
\text { resistance }\end{array}$ & $\begin{array}{c}\text { Fault detection result } \\
\text { and time }\left(\Delta t_{1}+\Delta t_{21}\right)\end{array}$ & $\begin{array}{l}\text { Faulty pole selection } \\
\text { result and time }\left(\Delta t_{1}+\Delta t_{2}\right)\end{array}$ \\
\hline \multirow{3}{*}{ P2P } & $0\left(f_{1}\right)$ & 0 & Internal fault in $3.01 \mathrm{~ms}$ & $\mathrm{P} 2 \mathrm{P}$ in $4.70 \mathrm{~ms}$ \\
\hline & $400 \mathrm{~km}\left(f_{2}\right)$ & $50 \Omega$ & Internal fault in $4.38 \mathrm{~ms}$ & $\mathrm{P} 2 \mathrm{P}$ in $6.07 \mathrm{~ms}$ \\
\hline & $800 \mathrm{~km}\left(f_{3}\right)$ & $100 \Omega$ & Internal fault in $3.03 \mathrm{~ms}$ & $\mathrm{P} 2 \mathrm{P}$ in $4.72 \mathrm{~ms}$ \\
\hline \multirow{3}{*}{$\mathrm{P} 2 \mathrm{G}$} & $0\left(f_{1}\right)$ & 0 & Internal fault in $3.01 \mathrm{~ms}$ & $\mathrm{P} 2 \mathrm{G}$ in $4.70 \mathrm{~ms}$ \\
\hline & $400 \mathrm{~km}\left(f_{2}\right)$ & $200 \Omega$ & Internal fault in $4.43 \mathrm{~ms}$ & $\mathrm{P} 2 \mathrm{G}$ in $6.12 \mathrm{~ms}$ \\
\hline & $800 \mathrm{~km}\left(f_{3}\right)$ & $500 \Omega$ & Internal fault in $5.65 \mathrm{~ms}$ & $\mathrm{P} 2 \mathrm{G}$ in $7.34 \mathrm{~ms}$ \\
\hline \multirow{3}{*}{$\mathrm{N} 2 \mathrm{G}$} & $0\left(f_{1}\right)$ & 0 & Internal fault in $3.01 \mathrm{~ms}$ & $\mathrm{~N} 2 \mathrm{G}$ in $4.70 \mathrm{~ms}$ \\
\hline & $400 \mathrm{~km}\left(f_{2}\right)$ & $200 \Omega$ & Internal fault in $4.83 \mathrm{~ms}$ & $\mathrm{~N} 2 \mathrm{G}$ in $6.52 \mathrm{~ms}$ \\
\hline & $800 \mathrm{~km}\left(f_{3}\right)$ & $500 \Omega$ & Internal fault in $5.67 \mathrm{~ms}$ & $\mathrm{~N} 2 \mathrm{G}$ in $7.36 \mathrm{~ms}$ \\
\hline \multirow{3}{*}{ P2G with $30 \mathrm{~dB}$ noise } & $0\left(f_{1}\right)$ & 0 & Internal fault in $3.03 \mathrm{~ms}$ & $\mathrm{P} 2 \mathrm{G}$ in $4.72 \mathrm{~ms}$ \\
\hline & $400 \mathrm{~km}\left(f_{2}\right)$ & $200 \Omega$ & Internal fault in $4.59 \mathrm{~ms}$ & $\mathrm{P} 2 \mathrm{G}$ in $6.28 \mathrm{~ms}$ \\
\hline & $800 \mathrm{~km}\left(f_{3}\right)$ & $500 \Omega$ & Internal fault in $5.67 \mathrm{~ms}$ & $\mathrm{P} 2 \mathrm{G}$ in $7.36 \mathrm{~ms}$ \\
\hline \multirow{3}{*}{$\begin{array}{l}\mathrm{N} 2 \mathrm{G} \text { with } 10 \% \text { parameter } \\
\text { error }\end{array}$} & $0\left(f_{1}\right)$ & 0 & Internal fault in $3.02 \mathrm{~ms}$ & $\mathrm{~N} 2 \mathrm{G}$ in $4.71 \mathrm{~ms}$ \\
\hline & $400 \mathrm{~km}\left(f_{2}\right)$ & $200 \Omega$ & Internal fault in $4.62 \mathrm{~ms}$ & $\mathrm{~N} 2 \mathrm{G}$ in $6.31 \mathrm{~ms}$ \\
\hline & $800 \mathrm{~km}\left(f_{3}\right)$ & $500 \Omega$ & Internal fault in $5.69 \mathrm{~ms}$ & $\mathrm{~N} 2 \mathrm{G}$ in $7.38 \mathrm{~ms}$ \\
\hline
\end{tabular}




\begin{tabular}{l|cccc} 
External nearby P2P with & $f_{4}$ & 0 & External fault & - \\
$30 \mathrm{~dB}$ noise & & $f_{4}$ & 0 & External fault \\
\hline $\begin{array}{l}\text { External nearby P2P with } \\
10 \% \text { parameter error }\end{array}$ & & & \\
\hline
\end{tabular}

According to the simulation results in Fig.10, Fig.11, Fig.12, and Table 2, based on full consideration of frequency dependence characteristics of transmission line and low-pass filter, accurate and rapid calculation of 1-mode current is realized in the novel current differential protection. By using the calculated 1-mode differential current as fault detection criterion, all kinds of internal faults could be detected within milliseconds, and no mal-operation could occur under any kind of transient process except internal faults. In addition, a faulty pole selection method is also proposed so that false trip of healthy pole could be avoided. The simulations have proved that on the premise of sensitivity, selectivity and reliability, the response time of novel current differential protection is only several milliseconds which is much shorter than hundreds of milliseconds of existing current differential protections. However, like all differential protections, the communication system and time synchronization system are essential, and time delay caused by communicating is an inevitable part of the total response time.

\section{Conclusion and expectation}

Protection of transmission lines is the most important defense against DC faults in a HVDC system. According to our studies in this paper, wave propagation of electrical quantities along line, caused by structure and intrinsic properties of transmission line, is the fundamental reason for the performance degradation of existing differential protections. Based on relations between line models and differential protection performances, a novel differential protection scheme, including fault detection and faulty pole selection by using mode current which only consists of low-frequency components is developed by us. Relative simulations of practical Wudongde $\pm 800 \mathrm{kV}$ demonstration projects in China are carried out to prove that novel differential protection could operate correctly to all faults within several milliseconds, which is much shorter than existing differential protections. Contributions of this paper are revealing the fundamental relation between line model and performance degradation and proposing a novel current differential protection which reduces the operation time from hundreds of milliseconds to several milliseconds.

The research results show the possible longest response time of novel differential protection for HVDC line with length of $800 \mathrm{~km}$ is $7.5 \mathrm{~ms}$, and it is still too long to completely avoid the block of VSC triggered by overcurrent. Studies on further improving operation speed of differential protection will be carried out in the future.

\section{Acknowledgements}

This work is supported by the State Key Laboratory of Advanced Power Transmission Technology (Grant No. GEIRI-SKL2019-001), the National Natural Science Foundation of China (Grant No. 51907141).

\section{References}

[1] Barnes M, Hertem DV, Teeuwsen SP, Callavik M. HVDC Systems in Smart Grids. Proceedings of the IEEE 2017;105:2082-98. https://doi.org/10.1109/JPROC.2017.2672879.

[2] Alassi A, Bañales S, Ellabban O, Adam G, MacIver C. HVDC Transmission: Technology Review, Market Trends and Future Outlook. Renewable and Sustainable Energy Reviews 2019;112:530-54. https://doi.org/10.1016/j.rser.2019.04.062.

[3] Korompili A, Wu Q, Zhao H. Review of VSC HVDC connection for offshore wind power integration. Renewable and Sustainable Energy Reviews 2016;59:1405-14. https://doi.org/10.1016/j.rser.2016.01.064. 
[4] Qin Y, Wen M, Zheng J, Bai Y. A novel distance protection scheme for HVDC lines based on R-L model. International Journal of Electrical Power \& Energy Systems 2018;100:167-77. https://doi.org/10.1016/j.ijepes.2018.02.037.

[5] Li J, Yang Q, Mu H, Le Blond S, He H. A new fault detection and fault location method for multi-terminal high voltage direct current of offshore wind farm. Applied Energy 2018;220:13-20. https://doi.org/10.1016/j.apenergy.2018.03.044.

[6] Chu X. Transient numerical calculation and differential protection algorithm for HVDC transmission lines based on a frequency-dependent parameter model. International Journal of Electrical Power \& Energy Systems 2019;108:107-16. https://doi.org/10.1016/j.ijepes.2018.12.039.

[7] Zhang Y, Li Y, Song J, Li B, Chen X. A New Protection Scheme for HVDC Transmission Lines Based on the Specific Frequency Current of DC Filter. IEEE Transactions on Power Delivery 2019;34:420-9. https://doi.org/10.1109/TPWRD.2018.2867737.

[8] Haleem NM, Rajapakse AD, Gole AM, Fernando IT. Investigation of Fault Ride-Through Capability of Hybrid VSC-LCC Multi-Terminal HVDC Transmission Systems. IEEE Transactions on Power Delivery 2019;34:241-50. https://doi.org/10.1109/TPWRD.2018.2868467.

[9] Zheng J, Wen M, Chen Y, Shao X. A novel differential protection scheme for HVDC transmission lines. International Journal of Electrical Power \& Energy Systems 2018;94:171-8. https://doi.org/10.1016/j.ijepes.2017.07.006.

[10] Zhang Y, Tai N, Xu B. Fault Analysis and Traveling-Wave Protection Scheme for Bipolar HVDC Lines. IEEE Transactions on Power Delivery 2012;27:1583-91. https://doi.org/10.1109/TPWRD.2012.2190528.

[11] Tong N, Lin X, Li Y, Hu Z, Jin N, Wei F, et al. Local Measurement-Based Ultra-High-Speed Main Protection for Long Distance VSC-MTDC. IEEE Transactions on Power Delivery 2019;34:353-64. https://doi.org/10.1109/TPWRD.2018.2868768.

[12] Lei A, Dong X, Shi S, Wang B, Terzija V. Equivalent traveling waves based current differential protection of EHV/UHV transmission lines. International Journal of Electrical Power \& Energy Systems 2018;97:282-9. https://doi.org/10.1016/j.ijepes.2017.11.017.

[13] Dai Z, Liu X, He Y, Huang M. Single-terminal Quantity Based Line Protection for Ring Flexible DC Distribution Grids. IEEE Transactions on Power Delivery 2019:1-1. https://doi.org/10.1109/TPWRD.2019.2920986.

[14] Song G, Chu X, Gao S, Kang X, Jiao Z. A New Whole-Line Quick-Action Protection Principle for HVDC Transmission Lines Using One-End Current. IEEE Transactions on Power Delivery 2015;30:599-607. https://doi.org/10.1109/TPWRD.2014.2300183.

[15] Gao S, Liu Q, Song G. Current differential protection principle of HVDC transmission system. IET Generation, Transmission \& Distribution 2017;11:128692. https://doi.org/10.1049/iet-gtd.2016.1380.

[16] Huai Q, Liu K, Qin L, Liao X, Zhu S, Li Y, et al. Backup-Protection Scheme for Multi-Terminal HVDC System Based on Wavelet-Packet-Energy Entropy. IEEE Access 2019;7:49790-803. https://doi.org/10.1109/ACCESS.2019.2910384.

[17] Chu X. Unbalanced current analysis and novel differential protection for HVDC transmission lines based on the distributed parameter model. Electric Power Systems Research 2019;171:105-15. https://doi.org/10.1016/j.epsr.2019.02.003.

[18] Guo W. The Principle and Analysis for the Incorrect Pickup of SIEMENS Electrode Bus Differential Protection. 2010 Asia-Pacific Power and Energy Engineering Conference, 2010, p. 1-5. https://doi.org/10.1109/APPEEC.2010.5449363.

[19] GLOVER JD, MULUKUTLA S. SARMA, THOMAS J. OVERBYE. Power System Analysis and Design, 5th edition. Cengage Learning; 2011.

[20] Li G, Liang J, Ma F, Ugalde-Loo CE, Liang H. Analysis of Single-Phase-to-Ground Faults at the Valve-Side of HB-MMCs in HVDC Systems. IEEE Transactions on Industrial Electronics 2018:1-1. https://doi.org/10.1109/TIE.2018.2829666.

[21] Li B, Liu H, Wen W, Cao H, Wang X, Lv H. DC Faults Ride-Through and Fast Recovery of MVDC system Based on Improved HB-MMC. IEEE J Emerg Sel Topics Power Electron 2019:1-1. https://doi.org/10.1109/JESTPE.2019.2920372.

[22] Hermann W.Dommol. electro magnetic transients program EMTP theory book. 1995.

[23] Martinez JA, Gustavsen B, Durbak D. Parameter determination for modeling system transients-Part I: overhead lines. IEEE Transactions on Power Delivery 2005;20:2038-44. https://doi.org/10.1109/TPWRD.2005.848678. 